Apidologie, 1978, 9 (3), 223-258.

\title{
ZUR MECHANIK DER KASTENENTSTEHUNG BEI DER HONIGBIENE (APIS MELLIFICA L.)
}

\section{Mécanisme de la formation des castes chez l'Abeille (Apis Mellifica $L$.)}

\author{
Karl WEISS \\ Bayerische Landesanstalt für Bienenzucht, \\ Burgbergstr. 70, D-8520 Erlangen
}

\section{SUMMARY}

\author{
MECHANISM OF THE CASTE FORMATION \\ IN THE HONEY BEE, APIS MELLIFICA L.
}

1. Female dimorphism of honeybee is based on exogenous influences in the way of different nourishment on larvae of the same genotype. Some time after hatching the larva possesses the capability of bipotent development. It means that a worker larva can be induced to become a typical queen and a queen larva on the other side to become a typical worker. Only the weight of the adults makes an exception in so far as being influenced in a caste-specific manner from the beginning of the larval life.

2. The bipotent (or sensitive) phase of worker larva which allows the larva to develop into the one or the other caste lasts till the third day of larval life. The transfer of older larvae to queen-cell cups yields to intermediates indicating a phase of undecided development (critical phase).

The bipotent phase of queen larva is shorter than that one of worker larva in respect of ovary development. If one transfers queen larvae $21 / 2$ days old to worker cells, one receives now and then imagines with an increased number of ovarioles. But the spermatheca is not influenced. Also the external characteristics (type of mandible and character of hind leg) become workerlike even though queen larvae are grafted in an age of three days or later. It seems that the critical phase for external features of queen larvae begins one day later than that one of worker larvae.

3. The critical phase of worker larva which creates intermediates is followed by a phase in which de velopment is determined in a distinct way not being changeable any more (fixed phase). The determina tion is settled after the fourth day of larval life.

The queen larva in the contrary is submitted to caste-determinant influences till near the end of the feeding period, forward the middle of the sixth larval day. The lack of food during this period yields to some deviations of external queenlike features in direction of a worker bee. Only the early established number of ovarioles does not vary any more. There is nearly no fixed phase.

4. In very rare cases, if one transfers worker larvae less than three days old to queen-cell cups one may get imagines which are no typical queens. Responsible for this is not the workerlike feeding during early lifespan but the deficiency of adequate care of the young larvae in an insufficient nurse colony. 


\section{ZUSAMMENFASSUNG}

1. Der weibliche Dimorphismus der Honigbiene gründet sich auf exogene, die Ernährung betreffende Einflüsse auf Larven des gleichen Genotyps. Diese Larven besitzen nach dem Schlüpfen eine Zeitlang die Fähigkeit zu bipotenter Entwicklung, d.h. eine Arbeiteriarve kann zur typischen Königin, eine Königinnenlarve zur typischen Arbeiterin umdeterminiert werden. Eine Ausnahme macht lediglich das Adultgewicht, welches vom ersten Larventag an kastentypisch beeinflusst wird.

2. Die bipotente oder sensible Phase mit voller Entwicklungsalternative in die eine oder andere Kaste währt bei der Arbeiterlarve bis zum Ende des 3. Lebenstages. Danach folgt eine Phase mit unentschiedener Entwicklung, während der bei der Umbettung von Arbeiterlarven in Weiselbecher mit königlicher Weiterpflege $Z$ wischentiere auftreten (kritische Phase).

Die bipotente Entwicklungszeit der Königinnenmade ist in Bezug auf die Ovarentwicklung kürzer. Arbeiterhafte Weiterpflege führte bereits bei der Umbettung von 2 1/2-tägigen Königinnenmaden in Arbeiterzellen vereinzelt zu einer Vermehrung der adulcen Ovariolenzahl. Die Samenblasen wurden dagegen nicht beeinflusst. Auch bezüglich der äusseren kastenspezifischen Merkmale Mandibelform und Ausbildung des Hinterbeins blieben die Larven von der anfangs königlichen Pflege - selbst bei Umbettung im Alter von über 3 Tagen nahezu unbeeinflusst. Für sie scheint die kritische Phase erst einen Tag später als bei der Arbeiterinnenlarve zu beginnen.

3. Bei der Arbeiterinnenmade folgt auf die kritische Phase mit unentschiedener Entwicklung eine fixierte Phase, in der sich an dem einmal eingeschlagenen Entwicklungsweg trotz königlicher Weiterpflege nichts mehr ändert. Die Determination ist nach dem 4. Larventag abgeschlossen.

Im Gegensatz dazu ist die Königinnenmade bis kurz vor Ende ihrer Fresszeit, um die Mitte des 6. Larventages, kastendeterminierenden Einflüssen unterworfen. Futtermangel hat zur Folge, dass Abweichungen von den äusseren königlichen Merkmalen in Richtung Arbeitsbiene auftreten. Lediglich an der frühzeitig angelegten Ovariolenzahl ändert sich nichts mehr. Die fixierte Phase fehlt fast ganz.

4. Es gibt seltene Ausnahmefälle, in denen auch schon aus jüngeren als 3-tägigen Arbeiterlarven nach der Ưbertragung in Weiselbecher Tiere mit mangelhaften königlichen Merkmalen entstehen können. Hierfür ist aber nicht die arbeiterhafte Vorernährung der Larven verantwortlich. Vielmehr sind sie in einem minderwertigen Pflegevolk mit unzulänglicher königlicher Fürsorge aufgezogen worden.

\section{I. - GEGENW}

Aus dem einheitlichen Genotyp des weiblichen Bieneneies gehen zwei deutlich verschiedene Phänotypen, die Arbeitsbiene und die Königin, hervor. Ausschlaggebend ist dabei die Ernährung während der Larvenzeit.

Die Arbeiterinnenmade ist durch Umbettung in eine Weiselwiege und Pflege in einem weisellosen Volk bis zu ihrem 3. Lebenstag zur Königin umstimmbar. ZANDER und BECKeR (1924) zählten die Eischläuche in den Ovarien, massen die Samenblase und beurteilten Mandibel- und Schlunddrüse der entstehenden Tiere. Sie konnten keine Anzeichen entdecken, dass die anfängliche Entwicklung als Arbeiterinnenlarve arbeiterähnliche Spuren bei den Imagines hinterlassen hätte. Werden die Larven jedoch während des 4 . Lebenstages umgebettet, macht sich die vorausgegangene Arbeiterentwicklung plötzlich im Organisationsbild der entstehenden Imago bemerkbar. Das konnten ECKERT (1934) für die Ovariolenzahlen und WEAVER (1957) für das reproduktive System, die Mandibeldrüsen und die äusseren Kastenmerkmale (Beschaffenheit von Metatarsus, Zungenlänge und Widerhakenzahl am Stachel) bestätigen. 
Abweichende Ergebnisse späterer Untersucher bedürfen der Kritik, wie WEISs (1971) aufzeigte. In seinen Versuchen entwickelten sich aus Arbeiterlarven bis zu 2 Tagen nach der Umbettung in Weiselwiegen weiselloser Völker merkmalsmässig vollkommene Königinnen. Lediglich das Adultgewicht und damit die Grösse der erwachsenen Tiere und ihrer Organe fällt aus diesem Rahmen. Das Adultgewicht ist schon vom frühesten Larvenstadium an beeinflussbar, indem es mit jedem Lebenstag der zur Aufzucht verwendeten Arbeiterlarven - zuerst geringfügig, dann immer deutlicher abnimmt (WEAVER 1959, JoRDAN 1960, WoyKe 1971, WeISS 1974).

Die Larven beider weiblicher Bienenkasten erhalten während ihrer ersten Lebenstage Futtersaft, der mengen- und inhaltsmässig kastenspezifische Unterschiede aufweist (zusf. Darst. bei Johansson u. Johansson 1958, Shuel u. Dixon 1960, Townsend u. Shuel 1962, Rembold 1964, HaydaK 1964). Dies hat nachweisbare Unterschiede im Stoffwechsel, im Hormonhaushalt und im Wachstum der jungen Larven zur Folge (siehe Lit. bei Shuel u. Dixon 1960, 1973, WeIss 1971, Rembold 1974), vermag aber andererseits keinen entscheidenden Einfluss auf die kastenspezifische Ausprägung der entstehenden Königinnen auszuüben. Biologisch ist das durchaus sinnvoll. Während normalerweise königliche Larven in Schwarm- oder Stillen Umweiselungszellen heranwachsen und von Anfang an mit königlichem Futter versorgt werden, ist dergleichen bei plötzlichem Ausfall der alten Stockmutter nicht möglich. Das Bienenvolk ist dann aber in der Lage, aus Arbeiterlarven Königinnen zu ziehen. Es entstehen über Arbeiterzellen sog. Nachschaffungszellen. Da den Bienen mit " halben " Königinnen wenig geholfen ist, ist es nur zweckmässig, wenn die Arbeitermaden relativ lange und möglichst vollkommen in die königliche Entwicklung umstimmbar bleiben. Wir sprechen von der bipotenten oder sensiblen Phase im Leben der Arbeiterlarve. Danach folgt eine Zeit der Unentschiedenheit in der Kastenausbildung, während der intermediäre Formen zwischen Königin und Arbeiterin entstehen.

Wenn die Arbeiterlarve während ihrer ersten Lebenszeit durch Änderung der kastenspezifischen Pflege auf den Entwicklungsweg einer Königin zu dirigieren ist, möchte man gerne wissen, ob das auch umgekehrt funktioniert. Kann auch die junge Königinnenlarve durch Umstellung ihrer Pflege zu einer Arbeitsbiene umgestimmt werden? In der Natur ist ein solcher Fall kaum vorgesehen. Er lässt sich aber experimentell nicht allzuschwer verwirklichen und es liegen auch schon einige orientierende Versuche von KLEIN (1904) und WEaver (1967) dazu vor. Sie deuten auf die Möglichkeit einer Umpolung der königlichen Entwicklung in Richtung Arbeitsbiene hin. Ich habe diese Versuche systematisch ausgebaut, wobei ich auf folgende Fragen besonderen Wert legte :

1. Vollzieht sich die Umstimmung der Königinnenmade in die Arbeiterkaste in derselben Weise wie umgekehrt die Umpolung der Arbeitermade in die königliche Entwicklung ?

2. Bis zu welchem Alter ist die Königinnenmade noch voli zur Arbeiterin umstimmbar? D.h. wann endet die sensible und wann beginnt die kritische Phase? 
3. Welcher Art sind die ersten Abweichungen von dem zunächst erwarteten voll kommenen Arbeitertyp?

Um den Zeitpunkt des Umschlagens der sensiblen Phase in die kritische Phase bei Arbeiter- und Königinnenlarven besser vergleichen zu können. hielt ich es für notwendig, noch einmal eine möglichst lückenlose Reihe von Umstimmungsversuchen, auch mit Arbeiterlarven verschiedenen Alters in die königliche Kaste, vorzunehmen. Ich wollte damit gleichzeitig aber noch eine andere Frage prüfen : Ist es richtig, dass im Anschluss an die kritische Entwicklungsphase der Arbeiterlarve, in der Zwischentiere entstehen, eine Phase folgt, in der die Larven ihre Sensibilität auf Futterumstellung völlig verlieren und in ihrer Entwicklung auf den bisher eingeschlagenen kastenspezifischen Weg unabweichbar festgelegt sind? Der kritischen Phase würde damit eine fixierte Phase in der Kastenentwicklung folgen.

Aus den Untersuchungen von BECKER (1925) geht hervor, dass über 4 Tage alte Arbeitermaden bei königlicher Weiterpflege reine Arbeiterinnen ergeben. Allerdings standen ihm nur 3 derartige Tiere zur Verfügung. Es ist offenbar nicht leicht, während der kritischen Determinationsphase in Weiselwiegen umgebettete Arbeitermaden zur Verpuppung und Imaginalhäutung zu bringen. Das geht auch aus Veröffentlichungen von ECKERT (1934), VAGT (1955), WEAVER (1957) und Woyke (1971) hervor. Diese Autoren haben erst gar nicht versucht, über 4 Tage alte Arbeiterlarven umzubetten, so dass eine Bestätigung des Arbeiterinnencharakters der Imagines, die aus so alten umgebetteten Arbeiterinnenmaden hervorgehen, noch aussteht. Ich habe mit der vorliegenden Arbeit diese Lücke zu schliessen versucht.

Wenn man sich für die kastenmässige Umstimmbarkeit älterer Arbeitermaden interessiert, möchte man auch umgekehrt gerne wissen, wie sich ältere Königinnenmaden in dieser Hinsicht verhalten. Der Umbettung von wesentlich über 3 Tage alten Königinnenmaden in Arbeiterzellen sind aber ebenfalls Grenzen gesetzt und zwar schon von der Madengrösse her. Uber 4 Tage alte Königinnenmaden bekommt man kaum mehr in die Arbeiterzellen hinein. Glücklicherweise gibt es einen anderen Weg, die Determinierbarkeit von Königinnenmaden in diesem fortgerückten Altersstadium zu untersuchen. Man lässt zu diesem Zweck decklungsreife Königinnenmaden am Ende ihrer durch Nahrungsaufnahme gekennzeichneten Entwicklungsperiode verschieden lange hungern.

Der erste Versuch dieser Art stammt von DönhofF (1859). Er nahm eine deckelungsreife Königinnenmade samt Futtersaft aus der Zelle und ersetzte sie durch eine andere Königinnenmade, die, nach seiner Schätzung, etwa 18 Stunden vor ihrer Verdeckelung stand. Dann verschloss er die Zelle mit Wachs. Mit 2 Tagen Verspätung schlüpfte eine besonders kleine Königin aus. VoN RHEIN ging 1933 systematisch vor. Er entfernte Königinnenmaden "vor der Reife" aus ihren Zellen und legte sie zur Verpuppung in kleine, mit Voile ausgekleidete Aluminiumschälchen. Die leichtesten Maden verpuppten sich nicht. Aus Larven mit einem Gewicht zwischen 162 und 180 mg erhielt er 3 Königinnen und 2 Puppen. Die kleinste Königin wog $91 \mathrm{mg}$ und war somit kleiner als eine normale Arbeiterin. Die Tiere waren königinnenhaft in der Aus- 
bildung ihrer Ovarien, der Mandibeln und der Hinterbeine. Nur die Samenblase schien nicht voll entwickelt. Bei zwei Tieren erinnerte die mehr dreieckige Kopfform an Verhältnisse bei der Arbeiterin. HAYDAK (1943) stellte in ähnlichen Versuchen eine hohe Sterblichkeit der heranwachsenden Königinnen fest. Er erhielt eine Arbeiterin und 8 normale Königinnen. 7 auf dem Puppenstadium eingegangene Tiere waren arbeiterähnlich. Die zur Arbeiterin tendierenden Individuen gingen aus Maden mit einem Durchschnittsgewicht von $204 \mathrm{mg}$ hervor, die Königinnen stammten aus Maden mit durchschnittlich $232 \mathrm{mg}$. Demgegenüber will SMITH (1959) aus weniger als $200 \mathrm{mg}$ schweren Larven, die er Königinnenzellen entnahm, in jeder Hinsicht königinnenhafte Imagines erhalten haben. Die umfangreichsten Versuche zu dieser Frage stellte JAY 1964 an. Er überführte je $10 \mathrm{Königinnenmaden} \mathrm{verschiedenen} \mathrm{Alters} \mathrm{in} \mathrm{vertikal} \mathrm{ge-}$ stellte Gelatinekapseln, die am unteren Ende mit Seidenpapier verschlossen wurden. Aus den leichtesten Larven der Gewichtskategorien 140 - $149 \mathrm{mg}$ und $160-169 \mathrm{mg}$ entstanden 2 Arbèiterinnen, aus den schwersten Larven mit 240 - $249 \mathrm{mg}, 280$ $289 \mathrm{mg}$ und über $320 \mathrm{mg}$ gingen 27 Königinnen hervor. Aus den Gewichtsklassen dazwischen (180 - $189 \mathrm{mg}, 200$ - $209 \mathrm{mg}$ ) entwickelten sich 2 Königinnen, 8 intermediäre Formen und 1 Arbeiterin. Die Beurteilung stützte sich auf die Ausbildung der Vorderkiefer und der Hinterbeine.

Nach dem vorliegenden Untersuchungsmaterial kann man also trotz einiger Widersprüche nicht daran zweifeln, dass die Königinnenmade auch nach dem 4. Larventag noch determinierbar ist. In den bisherigen Versuchen wurden keine Altersangaben von den Larven gemacht, so dass man in dieser Hinsicht auf Schätzungen aus dem Madengewicht angewiesen ist. Ich bemühte mich in einer Serie neuer Versuche diesem Mangel abzuhelfen. Es ging mir darum, zu erfahren, ob und wie lange die Königinnenlarve während ihrer Einspinnzeit, in der sie ja noch laufend Nahrung zu sich nimmt, hinsichtlich ihrer Kastenentwicklung beeinflussbar bleibt.

Zusammen mit den Erfahrungen bei der Umbettung von Königinnen- und Arbeiterlarven auf verschiedenem Entwicklungsstadium sollten die Versuche mit hungernden königlichen Altlarven genauere Einblicke in den allgemeinen Determinationsverlauf der weiblichen Bienenkasten geben. Dabei waren auch einige Aufschlüsse über den Grad der Empfindlichkeit einzelner Kastenmerkmale gegenüber den determinierenden Vorgängen zu erwarten.

\section{II. - METHODIK}

Der Fragestellung gemäss gliedert sich die Arbeit in 3 Teile :

1. Umstimmungsversuche mit Arbeiterinnenlarven

2. Umstimmungsversuche mit Königinnenlarven

3. Hungerversuche mit Königinnen-Altlarven

Entsprechend dieser Einteilung haben wir es auch mit 3 verschiedenen Praktiken bei der Beschaffung des Untersuchungsmaterials zu tun. Die Beurteilung der Versuchstiere geschieht jedoch nach einheitlichen Gesichtspunkten. 


\section{A. - Beschaffung des Versuchsmaterials}

\section{1. - Die königliche Aufzucht von Arbeiterlarven}

Zur Gewinnung vergleichbarer Königinnen verwendete ich in allen Versuchen Maden, die aus Zuchtvölkern der gleichen reinen Carnica-Linie stammten. Den Zuchtstoff genau datierten Alters erhielt ich durch Absperren der Königinnen von jeweils $8 \mathrm{Uhr}$ bis $12 \mathrm{Uhr}$, $12 \mathrm{Uhr}$ bis $20 \mathrm{Uhr}$ und $20 \mathrm{Uhr}$ bis $8 \mathrm{Uhr}$ auf bestimmten Bezirken leerer Waben. Ich verwendete dazu aufsteckbare Absperrgitter-Rahmen. Wenn die Maden nach einer bestimmten Zeit zum Umlarven verwendet wurden, waren sie auf 1/4 bzw. 1/2 Tag altersmässig genau festgelegt.

Die königliche Aufzucht fand in weisellosen Völkern statt, wobei die Zellen sofort nach dem Deckeln entfernt und zur Weiterentwicklung ihres Inhaltes in den Brutschrank $\left(35^{\circ} \mathrm{C}, 60 \%\right.$ r.F. $)$ verbracht wurden. Auf diese Weise waren mehrere aufeinanderfolgende Zuchtserien im gleichen Pflegevolk möglich.

Bei jedem Zuchtansatz legte ich die Arbeitermaden in angebrütete Weiselzellen auf Futtersaft, von dem ich unmittelbar vorher eine Königinnenmade gleichen Alters entfernt hatte. Dabei musste ich bei älteren Larven die Öffnung der Zelle durch Abschneiden des Zellenrandes erweitern. Durch die Umbettung auf Futtersaft sollte verhindert werden, dass in der Nahrungsaufnahme der Versuchsmaden eine Pause auftrat. In der Regel liess ich 3 verschiedene Altersstadien der Arbeitermaden nebeneinander im gleichen Pflegevolk aufziehen. Wenn mit einer schlechten Annahme zu rechnen war, erhielt das Pflegevolk nur Maden gleichen Alters.

Einen Tag vor der erwarteten Imaginalhäutung wurden die Puppen gewogen. Nach ihrer Fertigentwicklung zur Imago kamen sie bis zur präparativen Weiterverwendung in $70 \%$ igen Alkohol.

\section{2. - Die Aufzucht von Königinnenlarven in Arbeiterzellen}

Das notwendige königliche Larvenmaterial genau datierten Alters erhielt ich mit Hilfe der in Erlangen ausgearbeiteten Methode der "Zucht aus dem Ei » (WeIss 1962, 1975). Dabei wurde die Zuchtkönigin unter Absperrgitter auf eine unbebrütete $W$ abe gesetzt und nach 6 Stunden wieder befreit. Der Absperrgitter-Rahmen blieb auf der Wabe, bis diese nach 2 Tagen in kleine Abschnitte mit beidseitig 5-8 Zellen zerteilt wurde. Auf einer Seite enthielten die Zellen 2 - 21/4 tätige Eier. Mit der leeren Gegenseite zwickte ich die Zellgruppen in Klemmstopfen fest und befestigte diese am Zuchtrahmen. In den Zellnestern zerquirlte ich mit einem Streichholz alle überschüssigen Eier bis auf eines, um ein Zusammenwachsen mehrerer Weiselzellen zu verhindern. Die Zuchtrahmen kamen anschliessend in bestens vorbereitete weisellose Pflegevölker ohne offene Brut. So war eine königliche Aufzucht vom Ei ab gewährleistet.

Nach einer bestimmten, je nach Erfordernis des Versuchs verschiedenen Anbrütezeit nahm ich den Zuchtrahmen mit den inzwischen ausgebildeten Weiselkrügen heraus und bettete die königlichen Maden in Arbeiterzellen um. Sie wurden dabei mitten zwischen gleichaltrige Arbeiterbrut eingenistet. Zu diesem Zweck hatte ich die Stockmutter, welche die Eier für die Königinnenmaden lieferte, gleich nach der Entnahme aus dem Absperrgitterrahmen für einige Zeit auf eine leere Altwabe abgesperrt. Hier waren inzwischen Arbeitermaden geschlüpft, die nur um ein geringes jünger waren als die Königinnenmaden. Ich entfernte sie im Bereich eines kleinen Wabenviereckes und legte die Königinnenmaden an ihre Stelle. Durch Verwendung eines gefensterten Papiers, welches ich über die Wabe legte, konnte ich die einzelnen Versuchsareale später mühelos wierderfinden. Die Wabe übergab ich einem starken weiselrichtigen Volk zur Weiterpflege.

Nach dem Deckeln schnitt ich die Wabenstücke mit den Versuchsmaden heraus und brachte sie in kleinen Holzkästchen mit herausnehmbarer vorderer Glasscheibe, in sogenannten Okulierkäfigen, unter (Abb. 1). Gleichzeitig wurden Wabenbezirke mit Arbeiterbrut aus der Nachbarschaft gekäfigt. Beim Herausschneiden der Wabenstücke musste keine Brut zerstört werden, da die Schnittlinie bereits früher madenfrei gemacht worden war und die Rückseite der Wabe ohnehin keine Brut enthielt.

Die Okulierkäfige waren im hohlen Stopfen mit Futterteig versehen, enthielten aber kein Wasser, da eine unterschiedliche Wasseraufnahme der geschlüpften Tiere die Vergleichbarkeit der Schlupfgewichte 
beeinträchtig haben würde. Aus dem gleichen Grund achtete ich darauf, dass in den einzelnen brutfreien Zellen der Wabenstücke kein Honig oder Pollen lagerte, anderenfalls wurden solche Vorratszellen vorher sorgfältig entleert. Die Okulierkäfige kamen in den Brutschrank bei $35^{\circ} \mathrm{C}$ und $60 \%$ r.F. Ungefähr $1 \mathrm{Tag}$ nach dem Schlüpfen wurden Versuchs- und Kontrolltiere gewogen und in $70 \%$ igem Alkohol konserviert.

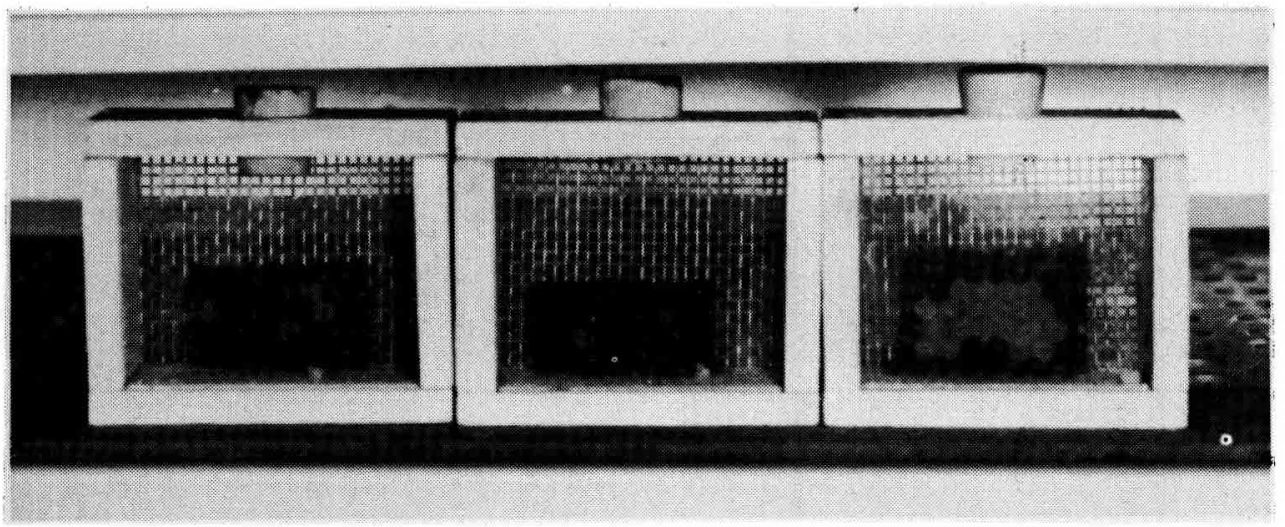

Aвв. 1. - Die Wabenstücke mit den umgebetteten Königinnenmaden kamen nach der Deckelung in Okulierkäfige und in den Brutschrank. In den ausgehöhlten Stopfen befand sich Invertinfutterteig für die Ertstversorgung der schlüpfenden Versuchs- und Krontrolltiere.

FIG. 1. - Les morceaux de rayon avec les larves de reine greffées sont mise après l'operculation dans des cages de greffage et à l'étuve. Dans les bouchons évidés est disposée une pâte de nourrissement à l'invertine pour la première alimentation des insectes testés et des témoins à éclore.

\section{3. - Hungerversuche mit königlichen Altlarven}

Eben geschlüpfte bis höchstens 1/4-tägige Arbeiterlarven wurden in künstliche Weiselbehälter umgebettet und nach gebräuchlicher Methode einem weisellosen Volk zur Pflege übergeben. Nach einer Entwicklungsdauer von mindestens 4 Tagen (vom Schlupf der Larve an gerechnet) nahm ich in Abständen von 6 Stunden jeweils einige Weiselzellen aus dem Pflegevolk. Ich befreite daraus die Larven und legte sie in kleine aus Stanniolpapier gefaltete Schiffchen. Nach der Bestimmung ihres Gewichtes wurden sic ein. zeln mitsamt ihren Stanniolgehäusen in praxisübliche Schlupfkäfige eingeschlossen und im Brutschrank bei $35^{\circ} \mathrm{C}$ und $60 \%$ r.F. untergebracht. Durch die Glastür des Brutschranks und die Zel!uleidfenster der Schlupfkäfige konnte ich die Vorgänge in den Stanniolbehältern gut beochachten. Soweit sich die Maden weiter entwickelten, wog ich die Puppen einen Tag vor der erwarteten Imaginalhäutung. Die fertigen Tiere wurden nach kurzem Aufenthalt in $70 \%$ igem Alkohol präpariert.

Zum Versuch diente mir die Zellenausbeute von 2 Zuchtserien, die gleichzeitig in 2 kräftigen Pflegevölkern durchgeführt worden ·waren.

\section{B. - Beurteilung der Kastenmerkmale}

Um die unter verschiedenen Gesichtspunkten erzeugten Versuchstiere im Hinblick auf ihre Kastenzugehörigkeit beurteilen zu können, verwendete ich Merkmale, die sich bei den beiden Kasten deutlich unterschieden und nach Möglichkeit auch noch messend oder zählend erfassen liessen. Neben dem Gewicht interessierte mich vor allem das reproduktive System (Ovariolenzahl und Samenblase), die Form des Kopfes, die Beschaffenheit der Vorderkiefer und die Ausgestaltung des 3. Beinpaares. Ich stellte die Widerhakenzahl des Stachels fest und ermittelte die Entwicklungszeit der Versuchstiere. 
Bei der Präparation trennte ich zuerst den Hinterleib von Rumpf. Von der hinteren Spitze beginnend durchschnitt ich mit einer feinen Schere den dorsalen Chitinpanzer des Abdomens bis zum vordersten Segment. Hier machte ich zwei kleine seitliche Einschnitte nach rechts und nach links. In der Wachsschale unter Wasser spreizte ich die beiden Integumenthälften auseinander und steckte sie mit Nadeln fest.

Bei der Königin liegen nun die beiden mächtigen Eierstöcke frei. Bei der Arbeitsbiene muss man die dünnen fadenförmigen Ovarien rechts und links des Darmes suchen. Die Ovariolenzahl der königlichen Eierstöcke wurde mit Hilfe der früher eingehend beschriebenen Technik unter dem Binokular ermittelt (WEIss 1971). Das Ovar der Arbeiterin wurde ohne Vorbehandlung auf einen Objektträger mit Hohlschliff gelegt. Die Ovariolen liessen sich mit feinsten Nadeln unter dem Binokular leicht auseinandertrennen und zählen (32-fache Vergr.).

Die Samenblase wurde noch in der Wasserschale mit dem Okularmikrometer bei ca. 50-facher Vergrösserung in medianer und lateraler Ausdehnung gemessen. Um einen mittleren Vergleichswert zu erhalten, summierte ich das Längen- und Breitenmass und teilte das Ergebnis durch zwei.
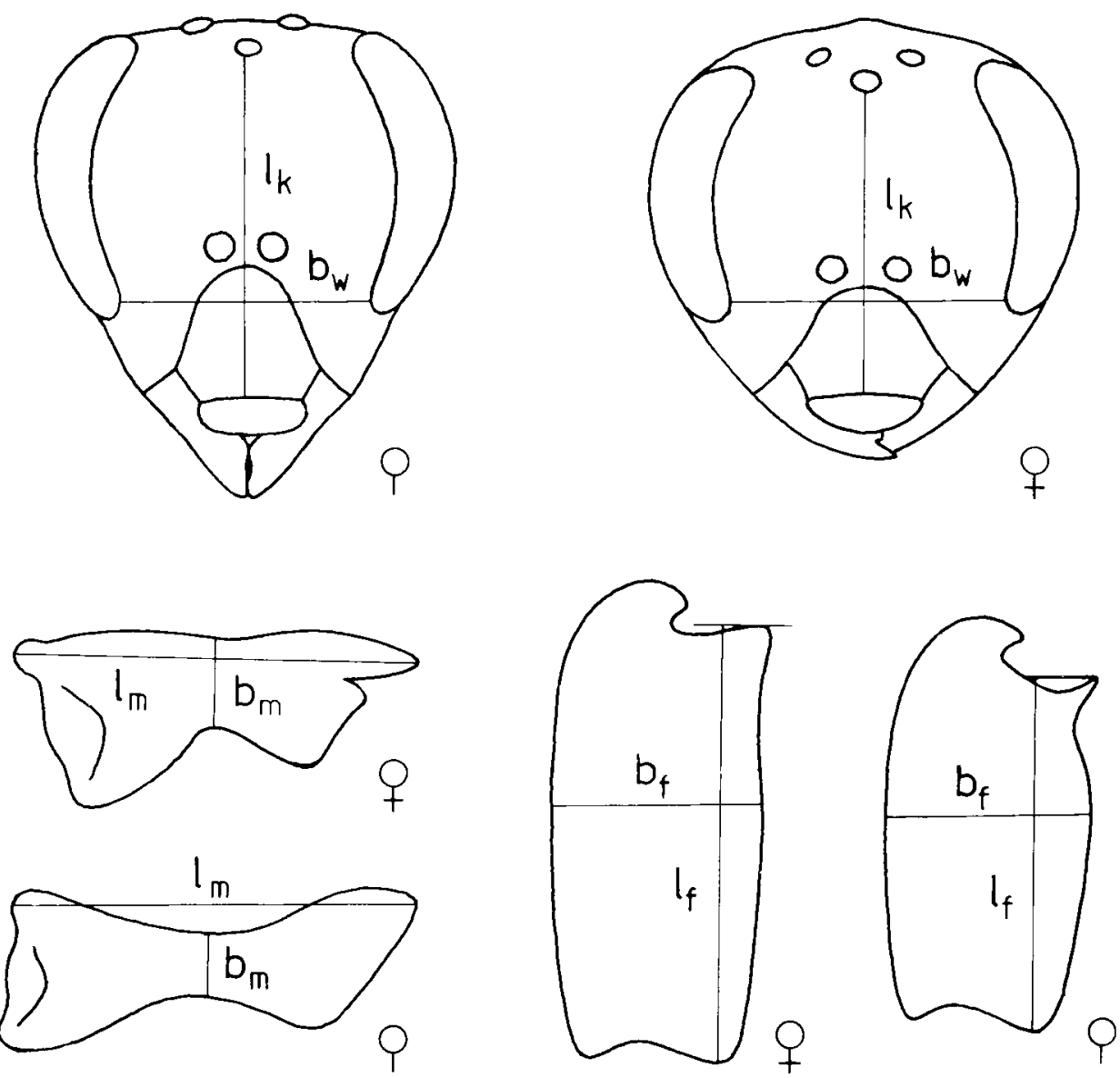

Aвв. 2. - In den schematischen Zeichnungen von Kopf, Mandibel und Ferse der waiblichen Bienenkasten sind die Messstrecken zur Indexbestimmung eingetragen.

FiG. 2. - Sur les dessins schématisés de la tête, de la mandibule et du tarse des castes femelles $\left(l_{k}\right)$ und stellt die kürzeste Entfernung zwischen den beiden Facettenaugen im Wangenbereich $\left(\mathrm{b}_{w}\right)$ fest. 
Zur Feststellung der Stachelhaken wurden die Stechborsten mit einer Pinzette am Stachelborstenbogen gefasst, von der quadratischen Platte getrennt und aus der Stachelrinne gezogen. Ich legte sie in etwas Wasser unter das Deckglas auf einen Objektträger und zählte die Widerhaken bei 170 -facher Vergrösserung unter dem Mikroskop. In der Regel befanden sich auf beiden Stechborsten gleichviel Stachelhaken.

Der Kopf der Arbeiterin gleicht, von vorne betrachtet, einem Dreieck. Bei der Königin ist er mehr rund. Um die Kopfform messend zu erfassen, trennt man den Kopf von der Brust ab und legt ihn bei 32facher Vergrösserung unter das Binokular. Man ermittelt mit Hilfe eines Okularmikrometers den Abstand zwischen dem unteren Rand des mittleren Stirnauges und dem freien medianen Ende des Kopfschildes $\left(b_{w}\right)$ und stellt die kürzeste Entfernung zwischen den beiden Fazettenaugen im Wangenbereich $\left(l_{R}\right)$ fest, Daraus errechnet man den Wangenindex $\frac{l_{k}}{b_{w}}$. Siehe hierzu Abb. 2.

Nach Abnahme der Kopfmasse wurde der Kopf zusammen mit den Hinterbeinen jedes Versuchstieres in einem offenen Gläschen zum Austrocknen im Zimmer aufbewahrt. Nach etwa 2 Wochen liessen sich die Mandibeln leicht von der Kopfkapsel absprengen. Ich legte die Kiefer mit der Innenseite nach oben auf einen Objektträger und umgab sie mit einer $0,3 \mathrm{~mm}$ starken Papiermaske, auf welche ich einen kleineren Objektträger zur Abdeckung legte. Bei 60 -facher Vergrösserung ermittelte ich die Entfernung von der Mandibelspitze bis zum ventralen Wurzelgelenk $\left(l_{m}\right)$ sowie die Breite der Mandibel an der engsten Stelle $\left(b_{m}\right)$. Daraus errechnete ich mit $\frac{l_{m}}{b_{m}}$ den Mandibelindex. Er ist bei der Königin (mit mehr gedrungenen Mandibeln) kleiner als bei der Arbeiterin, die einen schlankeren Oberkiefer besitzt.

Am getrockneten Hinterbein werden Tibia und Tarsus voneinander getrennt. Die Ferse der Königin ist lang und schmal, die der Arbeiterin kürzer und breiter. Ein zahlenmässiger Ausdruck dafür ist der Fersenindex $\frac{l_{\mathrm{f}}}{b_{\mathrm{f}}}$. welcher durch Division der Fersenlänge, gemessen vom freien distalen Ende der Ferse bis zum Pollenschieber $\left(l_{\mathfrak{f}}\right)$ durch die Fersenbreite an der bretesten Stelle $\left(b_{f}\right)$ gebildet wird. Bei den Messungen verwendet man zweckmässigerweise wieder eine Papiermaske zur Auflage für das abdeckende Objektträgerglas.

Mandibeln und Hinterbeine besitzen noch kastenspezifische Besonderheiten, die nicht zählend oder messend erfassbar sind. Gemeint ist der Mandibelzahn, welcher bei der Königin durch eine deutliche Einkerbung der Kaukante gebildet wird, bei der Arbeiterin hingegen völlig fehlt. Dafür besitzt das Hinterbein der Arbeiterin die typischen Pollensammeleinrichtungen der Bürste (an der medianen Seite des Metatarsus), des Pollenkamms (am distalen Ende der Tibia) und des Körbchens (einer kahlen schüsselförmigen Vertiefung in der äusseren Tibienfläche), wonach man bei der Königin vergeblich sucht. Nach einer bewährten Klasseneinteilung dieser Merkmale unterscheiden wir die kastentypischen Formen " $\mathrm{K}$ " und " $A$ ", sowie 3 Übergangsformen. Eine tendiert mehr zur Königin "ÜK ", eine mehr zur Arbeiterin "ÜA " und eine liegt etwa dazwischen "Ú ". Einzelheiten zur Charakterisierung dieser Ausbildungsformen der einzelnen Merkmale müssen bei WeIss (1975) nachgelesen werden.

Zur präparativen Aufarbeitung des Bienenmaterials halfen mir Frl. TsCHAKowsKy und Fr. Bosch, für deren wertvollen Einsatz ich mich hier bedanken möchte.

\section{III. - VERSUCHSERGEBNISSE}

\section{1. - Umstimmungsversuche mit verschieden alten Arbeiterlarven}

\section{a) Überblick}

In Tabelle 1 ist das Annahmeergebnis aller Zuchtversuche, bei denen Arbeiterlarven verschiedener Altersstufen in vorgepflegte Weiselzellen umgebettet wurden, zahlenmässig erfasst. Jede Zeile enthält eine Versuchsgruppe von bestimmtem Umlarvalter. Dabei entstammen die Versuchstiere jedes Umlarvalters in der Regel verschiedenen Pflegevölkern, was aus Rubrik 3 der Tabelle zu ersehen ist. 
TAB. 1. - Übersicht über die Umbettungsversuche mit Arbeiterlarven.

TABL. 1. - Tableau synoptique des expériences de transfert des larves d'ouvrière.

\begin{tabular}{|c|c|c|c|c|c|c|c|c|c|}
\hline 1 & 2 & 3 & 4 & \multicolumn{5}{|c|}{5} & 6 \\
\hline \multirow{2}{*}{$\begin{array}{l}\text { Nr. der } \\
\text { Vers. } \\
\text { gruppe }\end{array}$} & \multirow{2}{*}{$\begin{array}{l}\text { Alter der } \\
\text { umge- } \\
\text { betteten } \\
\text { Arb. } \\
\text { Larven }\end{array}$} & \multirow{2}{*}{$\begin{array}{c}\text { Zahl der } \\
\text { an der } \\
\text { Aufzucht } \\
\text { beteilig- } \\
\text { ten Pfle- } \\
\text { gevölker } \\
\text { Nombre des } \\
\text { colonies } \\
\text { éleveuses } \\
\text { ayant par- } \\
\text { ticipé à } \\
\text { l'élevage }\end{array}$} & \multirow{2}{*}{$\begin{array}{c}\text { Zahl der } \\
\text { umge- } \\
\text { betteten } \\
\text { Larven } \\
\text { Nombre } \\
\text { des larves } \\
\text { greffées }\end{array}$} & \multicolumn{5}{|c|}{$\begin{array}{l}\text { Larven erreichten das Entwicklungsstadium } \\
\text { Larves ayant atteint le stade de développement }\end{array}$} & \multirow{2}{*}{$\begin{array}{c}\text { Imagines } \\
\text { in \% } \\
\text { umge- } \\
\text { betteter } \\
\text { Larven } \\
\\
\text { Imagos } \\
\text { en \% de } \\
\text { larves } \\
\text { greffées }\end{array}$} \\
\hline & & & & $\begin{array}{l}\text { Nicht } \\
\text { angen. } \\
\text { Non } \\
\text { accepté }\end{array}$ & $\begin{array}{l}\text { Streck- } \\
\text { made } \\
\text { Prépupe } \\
1^{\text {er stade }}\end{array}$ & $\begin{array}{c}\text { Vor } \\
\text { puppe } \\
\text { Prépupe } \\
2^{\mathrm{e}} \text { stade }\end{array}$ & Puppe & Imago & \\
\hline 1 & $3 / 4-1$ & 1 & 20 & 5 & - & - & - & 15 & 75 \\
\hline 2 & $1-11 / 4$ & 4 & 30 & 8 & - & - & _. & 22 & 73 \\
\hline 3 & $11 / 4-13 / 4$ & 2 & 15 & - & - & . & - & 15 & 100 \\
\hline 4 & $13 / 4-2$ & 1 & 29 & 9 & - & - & - & 20 & 69 \\
\hline 5 & $\begin{array}{ll}2 & -21 / 4\end{array}$ & 2 & 40 & 10 & - & - & - & 30 & 75 \\
\hline 6 & $21 / 4-23 / 4$ & 3 & 39 & 12 & - & - & 3 & 24 & 62 \\
\hline 7 & $23 / 4-3$ & 2 & 39 & 18 & - & - & 1 & 20 & 51 \\
\hline 8 & $\begin{array}{ll}3 & -31 / 4\end{array}$ & 2 & 19 & 6 & - & - & 1 & 12 & 63 \\
\hline 9 & $31 / 4-33 / 4$ & 5 & 19 & 13 & 2 & - & 1 & 3 & 16 \\
\hline 10 & $33 / 4-4$ & 3 & 19 & 16 & - & - & - & 3 & 16 \\
\hline 11 & $4 \quad-41 / 4$ & 2 & 12 & 8 & - & 2 & - & 2 & 17 \\
\hline 12 & $41 / 4-43 / 4$ & 2 & 12 & 3 & - & - & - & 9 & 75 \\
\hline 13 & $43 / 4-5$ & 2 & 13 & 2 & 4 & 1 & - & 7 & 54 \\
\hline 14 & $\begin{array}{lll}5 & -5 & 1 / 4\end{array}$ & 3 & 16 & 9 & - & 1 & - & 8 & 50 \\
\hline
\end{tabular}

Beim Zahlenvergleich der umgebetteten Larven und den daraus entstandenen fertigen Tieren, deren prozentualer Anteil in der letzten Rubrik (6) wiedergegeben ist, stellt man fest, dass sich das Annahme- und Pflegeergebnis bei Verwendung von 3 1/4 bis 4 1/4- tägigen Arbeiterlarven gegenüber jüngeren Tieren deutlich verschlechtert. Es ist in diesem Zusammenhang interessant, aus den Versuchsprotokollen zu entnehmen, dass in manchen Zuchtversuchen mit Larven dieser Altersstadien überhaupt keine Annahme zustande kam und statt dessen die Pflegevölker " hoffnungslos weissellos " wurden. Schon von einem Larvenalter von 2 1/4 Tagen an treten öfter Sterbefälle bei älteren Entwicklungsstadien auf, welche bei der Verwendung jüngerer Maden nicht zu beobachten sind.

Die Quote fertig entwickelter Tiere nimmt bei einem Umlarvalter von 4 1/4 Tagen an wieder zu. Bei der Verwendung so alter Maden kam es gelegentlich vor, dass die Puppen umgekehrt, also mit dem Kopf zum Restfutter in der Zelle lagen.

Die Verwendung über 5 1/4 Tage alter Arbeiterlarven zu den Versuchen war uninteressant, da der Deckelungszeitpunkt der Arbeiterlarven zwischen 5 und 5 1/2 Tagen liegt und die Larven (im Gegensatz zu den Königinnenlarven) während des 
Einspinnens keine Nahrung mehr aufnehmen. Das dürfte auch für Arbeiterlarven zutreffen, die in diesem Alter noch in Weiselzellen umgebettet werden.

\section{b) Ergebnisse}

Alle wesentlichen Aussagen aus diesen Versuchen sind in Tabelle 2 und 3 wiedergegeben. Wie in der Übersichtstabelle umfasst jede Zeile eine Versuchsgruppe mit bestimmtem Umlarvalter. Wo die Kastenkriterien als Mittelwerte von Wäge-, Zähl-oder Messdaten (Indices) erscheinen, wurde eine Gegenüberstellung mit den Werten der Versuchsgruppe 1 vorgenommen, welche aufgrund des jungen Umlarvalters erwartungsgemäss vollkommen kastentypische Königinnen ergeben müsste. Wegen der uneinheitlichen Aufzuchtbedingungen der einzelnen Larvenalter in verschiedenen Pflegevölkern wurde zum Vergleich anstelle des üblichen t-Testes der parameterfreie Test von Mann und Whitney (WEBER 1963) herangezogen. Nur bei der Entwicklungsdauer musste ich von einer statistischen Bearbeitung absehen, da sich die Häutungskontrollen nicht immer in genügend kurzen und regelmässigen Zeitabständen durchführen liessen. Das würde irreführende Zahlenwerte ergeben haben.

Eine Zusammenschau der beiden Tabellen 2 und 3 lässt folgendes erkennen :

1. Der Einfluss der arbeitergemässen Erstversorgung der Larven auf den Phaenotyp der erwachsenen Tiere ist, was das Puppengewicht anbetrifft, schon bei der Umbettung relativ junger Larven zu erkennen (Tab. 2, Spalte 4).

2. Alle anderen untersuchten Kastenkriterien - Ovariolenzahl, Samenblasengrösse, Kopfform, Mandibelausbildung (Tab. 2, Spalte 5-9), Beschaffenheit von Ferse und Schiene des Hinterbeins, Stachelhaken und Entwicklungsdauer (Tab. 3, Spalte 49) - beginnen erst nach der Umbettung von über 3 Tage alten Arbeiterlarven arbeitermässig durchzuschlagen. Gelegentliche kleine Abweichungen in der Ausbildung der Kaukante der Mandibel können für sich allein noch nicht als Hinweis auf eine Kastenumstimmung gedeutet werden. Echte Zwischenformen in den Kastenkriterien treten erst bei der Umbettung von Arbeiterlarven zwischen dem 3. und 4. Lebenstag ein.

3. Eine Besonderheit zeigt die Versuchsgruppe Nr. 2. Überraschenderweise findet sich hier ein kleiner Prozentsatz von Tieren (2 Individuen) mit Abweichungen der Kaukante und der Beschaffenheit des Hinterbeins vom vollkommenen Weibchentyp ( Tab. 2, Spalte 9 u. Tab. 3, Spalte 5, 6, 7). Ein solcher Zwischentiercharakter, der sich vor allem noch im Gewicht und in der Grösse der Samenblase, aber bezeichnenderweise nicht in einer verminderten Ovariolenzahl bemerkbar machte, ist bei einem Umlarvalter von 1 - 1 1/4 Tagen normalerweise nicht zu erwarten. Die Tiere wurden in einem Volk aufgezogen, das äusserst pflegeunwillig war. Als Cordovan-Mutante nahm es trotz grosser Stärke nur ein paar wenige Zellen an. Ich hätte diesen verunglückten Versuch als Ausreisser überhaupt nicht mit in die tabellarische Aufbereitung der Versuchsergebnisse einzubeziehen brauchen, wenn er mir nicht gerade wegen der Abnormität seines Ausgangs aufschlussreich erschienen wäre ( $\mathrm{s}$. Diskussion).

4. Nach dem 4. Larventag ist die Arbeitermade durch königliche Weiterpflege in ihrer kastentypischen Ausbildung nicht mehr zu beeinflussen. Es entstehen vollkommene Arbeitsbienen. 
ТАВ. 2. - Ausbildung adulter Kastenmerkmale nach kö *** $p<0,001,{ }^{* *} p<0,01,{ }^{*} p<$

TABL. 2. - Formation des caractères adultes de caste après ${ }^{* * *} p<0,001$; $^{* *} p<0,01$ : $^{*} p<$

\begin{tabular}{|c|c|c|c|c|c|c|c|}
\hline 1 & 2 & 3 & \multicolumn{2}{|l|}{4} & \multicolumn{2}{|l|}{5} & \\
\hline $\begin{array}{c}\text { Nr. } \\
\text { der } \\
\text { Vers }\end{array}$ & $\begin{array}{c}\text { Alter der } \\
\text { umge- } \\
\text { betteten }\end{array}$ & $\begin{array}{c}\text { Zahl } \\
\text { der } \\
\text { Yers }\end{array}$ & \multicolumn{2}{|c|}{$\begin{array}{l}\text { Adultgewicht in } \mathrm{mg} \\
\text { Poids de l'adulte en } \mathrm{mg}\end{array}$} & \multicolumn{2}{|c|}{$\begin{array}{c}\text { Ovariolenzahl } \\
\text { Nombre dovarioles }\end{array}$} & $\begin{array}{l}\text { Samen } \\
\text { Diamètre }\end{array}$ \\
\hline $\begin{array}{l}N^{\circ} d u \\
\text { groupe } \\
\text { exp. }\end{array}$ & $\begin{array}{c}\text { Maden } \\
\text { Age des } \\
\text { larves d'o } \\
\text { greffées }\end{array}$ & $\begin{array}{c}\text { Nombre } \\
\text { d'insectes } \\
\text { en exp. }\end{array}$ & $\begin{array}{l}\bar{x} \\
\bar{x}\end{array}$ & $\begin{array}{l}\text { Diff. zu Gr. } 1 \\
\text { Diff./gr. } 1\end{array}$ & $\begin{array}{l}\bar{x} \\
\bar{x}\end{array}$ & $\begin{array}{l}\text { Diff. zu Gr. } 1 \\
\text { Diff./gr. } 1\end{array}$ & $\begin{array}{l}\text { in } \mathrm{T} . \mathrm{Str} \\
\bar{x} \\
\bar{x}\end{array}$ \\
\hline 1 & $3 / 4-1$ & 15 & $293,53 \pm 9,88$ & - & $343,20 \pm 12,04$ & - & $45.43 \pm$ \\
\hline 2 & $1-11 / 4$ & 22 & $277,23 \pm 39,80$ & $+16,30^{*}$ & $338,67 \pm 20,12$ & $+\quad 4.53^{-}$ & $44,58 \pm$ \\
\hline 3 & $11 / 4-13 / 4$ & 15 & $293,76 \pm 24,87$ & $-\quad 0,23^{-}$ & $338.67 \pm 34,54$ & $4,53^{-}$ & $46.05 \pm$ \\
\hline 4 & $13 / 4-2$ & 20 & $260,74 \pm 22,31$ & $+32,79^{* * *}$ & $348.37 \pm 14.39$ & $5.17^{-}$ & $45,16 \pm$ \\
\hline 5 & $\begin{array}{ll}2 & -21 / 4\end{array}$ & 30 & $246,47 \pm 52,42$ & $+47,06^{* *}$ & $339,83 \pm 15,73$ & $3,37^{-}$ & $43,97 \pm$ \\
\hline 6 & $21 / 4-23 / 4$ & 24 & $232,15 \pm 27,87$ & $+61,38^{* * *}$ & $339,88 \pm 18,99$ & $3.32^{-}$ & $44,19 \pm$ \\
\hline 7 & $23 / 4-3$ & 20 & $222,00 \pm 19,44$ & $+71,53^{* * *}$ & $334,80 \pm 16,05$ & $+\quad 8,40^{-}$ & $43,15 \pm$ \\
\hline 8 & $\begin{array}{lll}3 & -3 & 1 / 4\end{array}$ & 12 & $190,92 \pm 45,09$ & $+102,61^{* * *}$ & $241.50 \pm 89.76$ & $+101.70^{* * *}$ & $39,96 \pm 6$ \\
\hline 9 & $31 / 4-33 / 4$ & 3 & $119,00 \pm 23,39$ & $+174,53^{* * *}$ & $26,33 \pm 20,21$ & $+316,87^{* * *}$ & $12,00 \pm 10$ \\
\hline 10 & $33 / 4-4$ & 3 & $152,00 \pm 63,36$ & $+141,53^{*}$ & $54.00 \pm 57,30$ & $+289.20^{* * *}$ & $25,17 \pm 18$ \\
\hline 11 & $4 \quad-41 / 4$ & 2 & $112,00 \pm 7,07$ & $+181,53^{* * *}$ & $5,50 \pm 4,95$ & $+337,70^{* * *}$ & $5,00 \pm 0$ \\
\hline 12 & $41 / 4-43 / 4$ & 9 & $109,22 \pm 22,20$ & $+184,31^{* * *}$ & $6,57 \pm 3.69$ & $+336.63^{* * *}$ & $4,88 \pm$ \\
\hline 13 & $43 / 4-5$ & 7 & $132,14 \pm 8,88$ & $+161,39 * * *$ & $6.40 \pm 1.14$ & $+336,80^{* * *}$ & $5.50 \pm$ \\
\hline 14 & $\begin{array}{ll}5 & -51 / 4\end{array}$ & 8 & $125,75 \pm 16,45$ & $+167,78^{* * *}$ & $5,14 \pm 2,61$ & $+338,06^{* * *}$ & $5,29 \pm 0$ \\
\hline
\end{tabular}


$r$ Weiterpflege verschiedenen alter Arbeitermaden in vivo icht gesichert; Mann u. Whitney)

as royaux donnés in vivo à des larves d'ouvrières d'âge divers on significatif: Mann et Whitney).

\begin{tabular}{|c|c|c|c|c|c|c|c|c|c|}
\hline & \multicolumn{2}{|c|}{7} & \multicolumn{2}{|c|}{8} & \multicolumn{5}{|c|}{9} \\
\hline $\begin{array}{l}\text { iameter } \\
\text { mathèque }\end{array}$ & \multicolumn{2}{|c|}{$\begin{array}{l}\text { Wangenindex } \\
\text { Index jugulaire }\end{array}$} & \multicolumn{2}{|c|}{$\begin{array}{l}\text { Mandibelindex } \\
\text { Index mandibulaire }\end{array}$} & \multicolumn{5}{|c|}{$\begin{array}{c}\text { Kaukannte in Klassen-\% } \\
\text { Arêtes mandibulaires en \% classe }\end{array}$} \\
\hline $\begin{array}{l}\text { ff. zu Gr. } 1 \\
\text { Diff./gr. } 1\end{array}$ & $\begin{array}{l}\bar{x} \\
\bar{x}\end{array}$ & $\begin{array}{l}\text { Diff. zu Gr. } 1 \\
\text { Diff./gr. } 1\end{array}$ & $\begin{array}{l}\bar{x} \\
\bar{x}\end{array}$ & $\begin{array}{c}\text { Diff. zu Gr. } 1 \\
\text { Diff./gr. } 1\end{array}$ & $A$ & $\ddot{U} A$ & $\ddot{U}$ & $\ddot{U} K$ & $K$ \\
\hline $\begin{array}{c}-\overline{8} 5^{-} \\
0,62^{-} \\
0,27^{-} \\
1,46^{*} \\
1,24^{-} \\
2,28^{* *} \\
5,47^{* *} \\
33,43^{* * *} \\
20,26^{* * *} \\
40,43^{* * *} \\
40,55^{* * *} \\
39,93^{* * *} \\
40,14^{* * *}\end{array}$ & $\begin{array}{l}1,07 \pm 0,01 \\
1.06 \pm 0,02 \\
1,06 \pm 0,04 \\
1,06 \pm 0,03 \\
1,06 \pm 0,03 \\
1,07 \pm 0,03 \\
1,07 \pm 0,03 \\
1,12 \pm 0,05 \\
1,18 \pm 0,03 \\
1,16 \pm 0.03 \\
1,20 \pm 0,03 \\
1,21 \pm 0,01 \\
1,22 \pm 0,03 \\
1,22 \pm 0,06\end{array}$ & $\begin{array}{l}-\overline{0} \\
+0.01^{-} \\
+0.01^{-} \\
+0.01^{-} \\
+0.01^{-} \\
0 \\
0 \\
-0,05^{*} \\
-0,11^{* * *} \\
-0.09^{* * *} \\
-0.13^{* * *} \\
-0,14^{* * *} \\
-0,15^{* * *} \\
-0,15^{* * *}\end{array}$ & $\begin{array}{l}3,46 \pm 0,21 \\
3,59 \pm 0,30 \\
3,49 \pm 0,27 \\
3,44 \pm 0,16 \\
3,50 \pm 0,31 \\
3,53 \pm 0,21 \\
3,52 \pm 0,12 \\
4,00 \pm 0,46 \\
4,73 \pm 0,38 \\
4,43 \pm 0,51 \\
4,49 \pm 0,22 \\
4,34 \pm 0,41 \\
4,61 \pm 0,40 \\
4,77 \pm 0,29\end{array}$ & $\begin{array}{l}- \\
-0,13^{*} \\
-0,03^{-} \\
+0.02^{-} \\
-0.04^{-} \\
-0,07^{*} \\
-0.06^{-} \\
-0,54^{* * *} \\
-1,27^{* * *} \\
-0.97^{* * *} \\
-1.03^{* * *} \\
-0,88^{* * *} \\
-1,15^{* * *} \\
-1,31^{* * *}\end{array}$ & $\begin{array}{r}- \\
- \\
- \\
- \\
- \\
- \\
- \\
- \\
100 \\
67 \\
100 \\
100 \\
100 \\
100\end{array}$ & $\begin{array}{l}- \\
- \\
- \\
- \\
- \\
- \\
- \\
17 \\
- \\
- \\
- \\
- \\
- \\
-\end{array}$ & $\begin{array}{l}- \\
4,5 \\
- \\
- \\
- \\
- \\
- \\
- \\
- \\
33 \\
- \\
- \\
- \\
-\end{array}$ & $\begin{array}{l}\overline{4,5} \\
- \\
5 \\
- \\
4 \\
- \\
- \\
- \\
- \\
- \\
- \\
- \\
-\end{array}$ & $\begin{array}{r}100 \\
91 \\
100 \\
95 \\
100 \\
94 \\
100 \\
83 \\
- \\
- \\
- \\
- \\
- \\
-\end{array}$ \\
\hline
\end{tabular}


TAB. 3. - Ausbildung adulter Kastenkr alter Arbeiterma

TABL. 3. - Formation des caractères adu à des larves d'ouvrières $d$ '

\begin{tabular}{|c|c|c|c|c|c|c|c|c|c|}
\hline 1 & 2 & 3 & \multicolumn{2}{|c|}{4} & \multicolumn{5}{|c|}{5} \\
\hline \multirow{2}{*}{$\begin{array}{l}\text { Nr. der } \\
\text { Vers. Gr. } \\
\text { No du }^{\text {groupe }} \\
\text { exp. }\end{array}$} & \multirow{2}{*}{$\begin{array}{c}\text { Alter der } \\
\text { umgebetteten } \\
\text { Arb. Maden } \\
\text { Age des } \\
\text { larves d'o } \\
\text { greffées }\end{array}$} & \multirow{2}{*}{$\begin{array}{c}\text { Zahl der } \\
\text { Vers. Tiere } \\
\text { Nombre } \\
\text { d'insectes } \\
\text { en exp. }\end{array}$} & \multicolumn{2}{|c|}{$\begin{array}{l}\text { Fersenindex } \\
\text { Index tarsal }\end{array}$} & \multicolumn{5}{|c|}{$\begin{array}{c}\text { Bürste in Klassen- } \% \\
\text { Brosse à pollen en } \% \text { classe }\end{array}$} \\
\hline & & & $\begin{array}{l}\bar{x} \\
x\end{array}$ & $\begin{array}{c}\text { Diff. zu Gr. } 1 \\
\text { Diff./gr. } 1\end{array}$ & $A$ & $\ddot{U} A$ & $\ddot{U}$ & $\ddot{U} K$ & \\
\hline 1 & $3 / 4-1$ & 15 & $2,08 \pm 0,09$ & - & - & - & - & - & 1 \\
\hline 2 & $\begin{array}{lll}1 & -1 & 1 / 4\end{array}$ & 22 & $2,05 \pm 0,10$ & $+0,03^{-}$ & - & - & 4,5 & 4,5 & \\
\hline 3 & $11 / 4-13 / 4$ & 15 & $2,12 \pm 0,08$ & $-0,04^{-}$ & - & - & - & - & 1 \\
\hline 4 & $13 / 4-2$ & 20 & $2,05 \pm 0,09$ & $+0,03^{-}$ & - & - & - & - & i \\
\hline 5 & $2 \quad-21 / 4$ & 30 & $2,10 \pm 0,12$ & $-0,02^{-}$ & - & - & - & - & 1 \\
\hline 6 & $21 / 4-23 / 4$ & 24 & $2,09 \pm 0,09$ & $-0,01^{-}$ & - & - & - & - & 1 \\
\hline 7 & $23 / 4-3$ & 20 & $2,06 \pm 0,09$ & $+0,02$ & - & - & - & - & 1 \\
\hline 8 & $3 \quad-31 / 4$ & 12 & $1,93 \pm 0,12$ & $+0,15^{* * *}$ & - & 17 & 33 & - & \\
\hline 9 & $31 / 4-33 / 4$ & 3 & $1,75 \pm 0,07$ & $+0,33^{* * *}$ & 100 & - & - & - & \\
\hline 10 & $33 / 4-4$ & 3 & $1,81 \pm 0,10$ & $+0,27^{* * *}$ & 33 & 67 & - & - & \\
\hline 11 & $4 \quad-41 / 4$ & 2 & $1,70 \pm 0,04$ & $+0,38^{* * *}$ & 100 & - & - & - & \\
\hline 12 & $41 / 4-43 / 4$ & 9 & $1,69 \pm 0,08$ & $+0,39^{* * *}$ & 100 & - & - & - & \\
\hline 13 & $43 / 4-5$ & 7 & $1,81 \pm 0,09$ & $+0,27^{* * *}$ & 100 & - & - & - & \\
\hline 14 & $5 \quad-51 / 4$ & 8 & $1,75 \pm 0,06$ & $+0,33^{* * *}$ & 100 & - & - & - & \\
\hline
\end{tabular}


Ich habe ausserhalb der Tabellenangaben das Puppengewicht, die Zahlenwerte der Ovariolen, Stachelhaken und Entwicklungsdauer, sowie die Ausbildung und Indices von Kopf, Mandibel und Ferse der aus über 4 Tage alten Arbeiterinnen hervorgegangenen Versuchstiere mit den Kastenkriterien typischer Arbeiterinnen aus den Pflegevölkern verglichen. Es waren keinerlei Unterschiede festzustellen.

2. - Umstimmungsversuche mit verschieden alten Königinnenlarven

\section{a) Überblick}

Alle Versuche, in denen Königinnenmaden verschiedenen Alters (von $3 / 4$ bis 3 3/4 Tagen) in Arbeiterzellen zwischen gleichaltrige Arbeiterbrut weiselrichtiger Völker umgebettet wurden, sind in Tabelle 4 erfasst. Jede Altersgruppe von Königinnenlarven stammte jeweils aus nur einem Pflegevolk und wurde zur gleichen Zeit und unter gleichen Bedingungen umgebettet.

ТАВ. 4. - Übersicht über die Umbettungsversuche mit Königinnenmaden.

TABL. 4. - Tableau synoplique des expériences de transferl de larves de reines.

\begin{tabular}{|c|c|c|c|c|c|}
\hline 1 & 2 & 3 & 4 & 5 & 6 \\
\hline $\begin{array}{l}\text { Vers. Nr. } \\
\text { Exp. no }\end{array}$ & $\begin{array}{l}\text { Alter der } \\
\text { umgebetteten } \\
\text { Kö. Maden } \\
\text { Age des } \\
\text { larves } \\
\text { greffees }\end{array}$ & $\begin{array}{l}\text { Zahl der } \\
\text { umgebetteten } \\
\text { Larven } \\
\\
\text { Nombre de } \\
\text { larves greffées }\end{array}$ & $\begin{array}{c}\text { Zahl der } \\
\text { fertig } \\
\text { entwickelten } \\
\text { Tiere } \\
\text { Nombre dinsectes } \\
\text { entièrement } \\
\text { developpés }\end{array}$ & $\begin{array}{c}\text { Imagines in \% } \\
\text { umgebetteter } \\
\text { Tiere } \\
\text { Imagos en } \% \text { des } \\
\text { larves greffees }\end{array}$ & $\begin{array}{c}\text { Zahl der } \\
\text { Vergl. Arb. } \\
\text { Nombre des } \\
\text { comparées }\end{array}$ \\
\hline $\begin{array}{r}1 \\
2 \\
3 \\
4 \\
5 \\
6 \\
7 \\
8 \\
9 \\
10 \\
11 \\
12 \\
13 \\
14 \\
15 \\
16 \\
17 \\
18 \\
19 \\
20\end{array}$ & 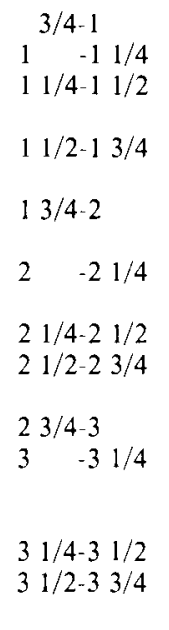 & $\begin{array}{l}22 \\
25 \\
16 \\
31 \\
25 \\
30 \\
31 \\
22 \\
23 \\
24 \\
11 \\
19 \\
17 \\
15 \\
19 \\
23 \\
20 \\
10 \\
23 \\
27\end{array}$ & $\begin{array}{r}20 \\
22 \\
10 \\
28 \\
19 \\
22 \\
21 \\
18 \\
16 \\
16 \\
9 \\
17 \\
14 \\
14 \\
13 \\
4 \\
11 \\
3 \\
3 \\
0\end{array}$ & $\begin{array}{r}91 \\
88 \\
63 \\
90 \\
76 \\
73 \\
68 \\
82 \\
70 \\
67 \\
82 \\
89 \\
82 \\
93 \\
68 \\
17 \\
55 \\
30 \\
13 \\
0\end{array}$ & $\begin{array}{l}24 \\
26 \\
24 \\
27 \\
17 \\
21 \\
27 \\
27 \\
17 \\
21 \\
24 \\
26 \\
24 \\
22 \\
21 \\
19 \\
19 \\
19 \\
22 \\
21\end{array}$ \\
\hline
\end{tabular}


Im Verfolg der Spalte 5 kann man ein relativ gutes Pflegeergebnis der Maden im Umlarvalter bis zu etwa 3 Tagen feststellen. Darauf geht der Annahmeprozentsatz rasch zurück. So haben sich in 3 Versuchen mit 62 übertragenen 3 - 31 1/4-tägigen Königinnenmaden nur noch $28(45 \%)$ adulte Tiere entwickelt, aus einem Versuch mit 10 $31 / 4$ - 3 1/2-tägigen Maden gingen $3(30 \%)$ und aus zwei weiteren mit 50 Maden ebenfalls nur $3(6 \%)$ fertig ausgebildete Individuen hervor. Noch ältere Larven nahmen die Bienen in meinen Versuchen überhaupt nicht mehr an. Zweifellos war es nicht ganz leicht, die grossen Larven in die engen Arbeiterzellen umzubetten, aber der totale Annahmeausfall hat sicherlich nichts mit der Möglichkeit einer mechanischen Beschädigung der Larven zu tun.

$\mathrm{Zu}$ jeder Gruppe von Umbettungstieren gehört eine bestimmte Vergleichsgruppe von Arbeiterinnen, die sich in der Nachbarschaft der umgebetteten Königinnenlarven ganz regulär aus Arbeitermaden entwickelt haben (Spalte 6).

\section{b) Ergebnisse}

In den Tabellen 5 und 6 werden die Kastenmerkmale der Tiere, welche aus der Übertragung von verschieden alten Königinnenlarven in Arbeiterzellen entstanden, mit denen der reinen Arbeiterinnen verglichen, die jeweils aus benachbarten Brutbezirken hervorgingen. Da jede Zeile einen geschlossenen Versuchsansatz darstellt, konnte das Zahlenmaterial statistisch mit dem t-Test überprüft werden. Bei der Entwicklungsdauer unterblieb die statistische Bearbeitung aus den vorne genannten Gründen.

1. Im Hinblick auf die Gewichte - sowohl der Versuchs- als auch der Vergleichstiere - kann man grosse Schwankungen zwischen den Versuchen feststellen (Tab. 5, Spalte 5). Versuchs- und Vergleichsgruppe variieren dabei meist gleichsinnig. Ein Grund hierfür sind sicherlich die zur Larvenaufzucht verwendeten Waben, die einmal heller, einmal dunkler, d.h. verschieden oft bebrütet waren. Je mehr Brutgenerationen in einer Wabe aufgezogen werden, desto enger werden die mit Kokonhäuten austapezierten Zellen und desto kleiner geraten die daraus ausschlüpfenden Bienen. Ich musste die bebrüteten Waben zu den Versuchen verwenden, weil sie robuster sind als unbebrütete. Infolgedessen liessen sich die Königinnenmaden leichter in die Arbeiterzellen umbetten, und auch der spätere Umgang mit den Wabenstücken war erleichtert. Über den Einfluss der Wabe hinaus können die Gewichtsschwankungen von Zeile zu Zeile auch noch auf Unterschiede in der Pflegeintensität der Ammen zurückzuführen sein. Bekanntlich wird die Brut in verschiedenen Völkern und zu verschiedenen Zeiten unterschiedlich gut versorgt.

2. $\mathrm{Zu}$ den Gewichtsschwankungen zwischen den Versuchen kommen Unterschiede innerhalb der Versuche. Dabei wogen die Versuchstiere im Mittel schwerer als die Vergleichsarbeiterinnen. Ein Blick auf die häufigen Plus-Zeichen in der Spalte mit den Differenzwerten und deren gelegentliche statistische Sicherung weist das deutlich aus. Überraschenderweise treten diese Gewichtsunterschiede der adulten Tiere nicht erst bei der Umbettung älterer Königinnenmaden auf, sondern schon bei der Verwendung sehr junger Larven. Mit dem parameterfreien Test von Mann und Whitney ( $\mathrm{s}$.W. 
TAB. 5. - Ausbildung adulter Kastenmerkmale nach weiselrichtiger Völker im Vergleich mit reinen Arb (*** $p<0,001 ;{ }^{* *} p<0,01$;

TABL. 5. - Formation des caractères adultes $d$ dans des cellules d'ouvrières de colonies orphelines provenant de portions de couvain voisines

\begin{tabular}{|c|c|c|c|c|c|c|c|}
\hline 1 & 2 & 3 & 4 & & 5 & & \\
\hline \multirow[b]{2}{*}{$\begin{array}{l}\text { Vers. } \\
\text { Nr. } \\
\\
\text { Exp. } \\
N^{\circ}\end{array}$} & \multirow{2}{*}{$\begin{array}{c}\text { Alter der } \\
\text { umgebette- } \\
\text { ten König. } \\
\text { Maden } \\
\text { Age des } \\
\text { larves de } \\
\text { greffées }\end{array}$} & \multirow[b]{2}{*}{$\begin{array}{c}\text { Vers. } \\
\text { Tiere } \\
\mathrm{n}_{\mathrm{a}} \\
\text { Insectes } \\
\text { testés } \\
\mathrm{n}_{a}\end{array}$} & \multirow[b]{2}{*}{$\begin{array}{c}\text { Vergl. } \\
\text { Arb. } \\
\mathrm{n}_{\mathrm{b}} \\
\text { Ouvrières } \\
\text { comparées } \\
\mathrm{n}_{b}\end{array}$} & \multicolumn{3}{|c|}{$\begin{array}{l}\text { Adultgewicht in } \mathrm{mg} \\
\text { Poids des adultes en } \mathrm{mg}\end{array}$} & \\
\hline & & & & $\begin{array}{l}\text { Vers. Tiere } \\
\bar{x} \\
\text { Ins. testés }\end{array}$ & $\begin{array}{l}\text { Vergl. Arb. } \\
\bar{x} \quad s \\
{ }_{\bar{x}}^{\text {compar. }}\end{array}$ & $\begin{array}{c}\text { Differenz } \\
a-b \\
\\
\text { Diff. } \\
a-b\end{array}$ & $\begin{array}{cc} & \text { Vers. Tiere } \\
\bar{x} & \text { (Min-Max) } \\
& \\
& \text { Ins. testés } \\
\bar{x} & (\text { Min-Max })\end{array}$ \\
\hline 1 & $3 / 4-1$ & 20 & 24 & $96.25+5.91$ & $91,00+4,42$ & $+5,25^{* *}$ & $7,50(2-17) \pm 4$ \\
\hline 2 & $1 \quad-11 / 4$ & 22 & 26 & $86,29 \pm 5,89$ & $84,19 \pm 7,33$ & $+2,10^{-}$ & $8,20(3-15) \pm$ \\
\hline 3 & $11 / 4-11 / 2$ & 10 & 24 & $95,90 \pm 3,84$ & $91,00 \pm 4,42$ & $+4,90^{* *}$ & $8,30(3-12) \pm$ \\
\hline 4 & & 28 & 27 & $95,61 \pm 5,86$ & $97,78 \pm 7,88$ & $-2,17^{-}$ & $7,88(3-21) \pm$ \\
\hline 5 & $11 / 2-13 / 4$ & 19 & 27 & $91,32 \pm 5,28$ & $90,56 \pm 6,43$ & $+0,76^{-}$ & $8,11(3-15) \pm$ \\
\hline 6 & & 22 & 21 & $91,55 \pm 6,05$ & $85,86 \pm 6,51$ & $+5,69^{* *}$ & $8,05(3-13) \pm$ \\
\hline 7 & $13 / 4-2$ & 21 & 27 & $100,75 \pm 8,31$ & $90,56 \pm 6,43$ & $+10,19^{* * *}$ & $8,63(2-16) \pm$ \\
\hline 8 & & 18 & 27 & $100,50 \pm 3,45$ & $97,78 \pm 7,88$ & $+2,72^{-}$ & $7,29(3-17) \pm$ \\
\hline 9 & $-21 / 4$ & 16 & 17 & $86,50 \pm 8,16$ & $88,00 \pm 3,86$ & $-1,50^{-}$ & $6,00(4-8) \pm$ \\
\hline 10 & & 16 & 21 & $96,18 \pm 7,48$ & $85,86 \pm 6,51$ & $+10,32^{* * *}$ & $10,47(3-34) \pm 8$ \\
\hline 11 & $21 / 4-21 / 2$ & 9 & 24 & $86,44 \pm 7,84$ & $91,00 \pm 4,42$ & $-4,56^{-}$ & $15,22(4-70) \pm 20$ \\
\hline 12 & $21 / 2-23 / 4$ & 17 & 26 & $90,47 \pm 7,59$ & $84,19 \pm 7,33$ & $+6,28^{*}$ & $9,00(2-16) \pm 4$ \\
\hline 13 & & 14 & 24 & $93,36 \pm 4,58$ & $91,00 \pm 4,42$ & $+2,36^{-}$ & $23,86(3-130) \pm 37$ \\
\hline 14 & $23 / 4-3$ & 14 & 22 & $93,54 \pm 4,72$ & $89,09 \pm 6,38$ & $+4,45^{-}$ & $11,00(3-39) \pm 9$ \\
\hline 15 & $\begin{array}{lll}3 & -3 & 1 / 4\end{array}$ & 13 & 21 & $82,92 \pm 8,61$ & $85,86 \pm 6,51$ & $-2,94^{-}$ & $54,31(3-199) \pm 64$ \\
\hline 16 & & 4 & 19 & $91,75 \pm 4,50$ & $90,21 \pm 4,34$ & $+1,54^{-}$ & $32,67(11-66) \pm 29$ \\
\hline 17 & & 11 & 19 & $105,30 \pm 15,40$ & $90,21 \pm 4,34$ & $+15,09^{* *}$ & $45,00(2-260) \pm 81$ \\
\hline 18 & $31 / 4-31 / 2$ & 3 & 19 & $98,00 \pm 19,98$ & $90,21 \pm 4,34$ & $+7,79^{-}$ & $21,00(5-52) \pm 26$ \\
\hline 19 & $31 / 2-33 / 4$ & 3 & 22 & $84,00 \pm 14,80$ & $89,09 \pm 6,38$ & $-5,09^{-}$ & $35,00(2-91) \pm 48$ \\
\hline
\end{tabular}


ung verschieden alter Königinnenlarven in Arbeiterzellen nen gleicher Herkunft aus benachbarten Brutbezirken 0,05; - nicht gesichert; t-Test).

après transfert de larves de reines d'âge variable paraison avec des ouvrières pures de la même origine $1 ;{ }^{* *} p<0,01 ;^{*} p<0,05 ;{ }^{-}$non significatif; test-t).

\begin{tabular}{|c|c|c|c|c|c|c|c|}
\hline & \multicolumn{3}{|c|}{7} & \multicolumn{3}{|c|}{8} \\
\hline \multicolumn{2}{|l|}{$\begin{array}{l}\text { olenzahl } \\
\text { d'ovarioles }\end{array}$} & \multicolumn{3}{|c|}{$\begin{array}{l}\text { Samenblasen-Diameter in T. Str. } \\
\text { Diamètre de la Spermathèque }\end{array}$} & \multicolumn{3}{|c|}{$\begin{array}{l}\text { Wangenindex } \\
\text { Index jugulaire }\end{array}$} \\
\hline $\begin{array}{c}\text { Vergl. Arb. } \\
\bar{x} \text { (Min-Max) } s \\
\\
\bar{x}(\text { Compar. } \\
\end{array}$ & $\begin{array}{c}\text { Differenz } \\
a-b \\
\text { Diff. } \\
a-b\end{array}$ & $\begin{array}{c}\text { Vers. Tiere } \\
\begin{array}{l}\bar{x} \\
\text { Ins. testés } \\
\bar{x} \quad s\end{array}\end{array}$ & $\begin{array}{l}\text { Vergl. Arb. } \\
\begin{array}{l}\bar{x} \\
P_{\bar{x}}\end{array} \\
\text { compar. }\end{array}$ & $\begin{array}{c}\text { Differenz } \\
a-b \\
\text { Diff. } \\
a-b\end{array}$ & $\begin{array}{l}\text { Vers. Tiere } \\
\bar{x} \quad s \\
\text { Ins. testés } \\
\bar{x} \quad s\end{array}$ & $\begin{array}{l}\text { Vergl. Arb. } \\
\bar{x} \quad s \\
\underbrace{\text { compar. }}_{x}\end{array}$ & $\begin{array}{l}\text { Diffe- } \\
\text { renz } \\
a-b \\
\text { Diff. } \\
a-b\end{array}$ \\
\hline $\begin{array}{l}, 74(3-18) \pm 3,88 \\
, 30(2-12) \pm 2,91 \\
, 74(3-18) \pm 3,88 \\
, 96(3-15) \pm 2,93 \\
, 00(2-8) \pm 2,55 \\
, 00(3-11) \pm 2,75 \\
, 00(2-8) \pm 2,55 \\
, 96(3-15) \pm 2,93 \\
, 88(3-10) \pm 2,30 \\
, 00(3-11) \pm 2,75 \\
, 74(3-18) \pm 3,88 \\
, 30(2-12) \pm 2,91 \\
, 74(3-18) \pm 3,88 \\
, 50(3-15) \pm 3,65 \\
, 00(3-11) \pm 2,75 \\
, 89(2-10) \pm 2,08 \\
, 89(2-10) \pm 2,08 \\
, 89(2-10) \pm 2,08 \\
, 50(3-10) \pm 3,65\end{array}$ & $\begin{array}{l}-0,24^{-} \\
+1,90^{-} \\
+\quad 0,56^{-} \\
+1,92^{-} \\
+1,11^{-} \\
+1,05^{-} \\
+1,63^{-} \\
+1,33^{-} \\
+0,12^{-} \\
+3,47^{-} \\
+7,48^{-} \\
+2,70^{-} \\
+16,12^{*} \\
+3,50^{-} \\
+47,31^{* *} \\
+26,78^{* *} \\
+39,11^{* * *} \\
+15,11^{* *} \\
+27,50^{* *}\end{array}$ & $\begin{array}{l}5,31 \pm 0,43 \\
4,92 \pm 0,65 \\
5,46 \pm 0,67 \\
5,23 \pm 0,33 \\
5,01 \pm 0,48 \\
4,99 \pm 0,50 \\
5,12 \pm 0,72 \\
5,10 \pm 0,38 \\
4,98 \pm 0,43 \\
5,37 \pm 0,47 \\
5,20 \pm 0,71 \\
5,30 \pm 0,47 \\
5,36 \pm 0,71 \\
4,98 \pm 0,53 \\
5,40 \pm 0,66 \\
5,00 \pm 0,34 \\
6,11 \pm 0,64 \\
5,32 \pm 0,28 \\
5,61 \pm 0,36\end{array}$ & $\begin{array}{l}5,26 \pm 0,34 \\
5,11 \pm 0,27 \\
5,26 \pm 0,34 \\
4,99 \pm 0,60 \\
5,25 \pm 0,55 \\
5,05 \pm 0,52 \\
5,25 \pm 0,55 \\
4,99 \pm 0,60 \\
5,22 \pm 0,71 \\
5,05 \pm 0,52 \\
5,26 \pm 0,34 \\
5,11 \pm 0,27 \\
5,26 \pm 0,34 \\
5,20 \pm 0,70 \\
5,05 \pm 0,52 \\
5,22 \pm 0,80 \\
5,22 \pm 0,80 \\
5,22 \pm 0,80 \\
5,20 \pm 0,70\end{array}$ & $\begin{array}{l}+0,05^{-} \\
-0,19^{-} \\
+0,20^{-} \\
+0,24^{-} \\
-0,24^{-} \\
-0,06^{-} \\
-0,13^{-} \\
+0,11^{-} \\
-0,24^{-} \\
+0,32^{-} \\
-0,06^{-} \\
+0,19^{-} \\
+0,10^{-} \\
-0,22^{-} \\
+0,35^{-} \\
-0,22^{-} \\
+0,89^{* *} \\
+0,10^{-} \\
+0,41^{-}\end{array}$ & $\begin{array}{l}1,26 \pm 0,02 \\
1,27 \pm 0,04 \\
1,24 \pm 0,01 \\
1,25 \pm 0,02 \\
1,26 \pm 0,06 \\
1,25 \pm 0,03 \\
1,27 \pm 0,03 \\
1,24 \pm 0,02 \\
1,25 \pm 0,02 \\
1,26 \pm 0,02 \\
1,25 \pm 0,02 \\
1,24 \pm 0,04 \\
1,24 \pm 0,02 \\
1,25 \pm 0,02 \\
1,25 \pm 0,02 \\
1,25 \pm 0,02 \\
1,26 \pm 0,03 \\
1,25 \pm 0,01 \\
1,27 \pm 0,01\end{array}$ & $\begin{array}{l}1,26 \pm 0,02 \\
1,26 \pm 0,03 \\
1,26 \pm 0,02 \\
1,25 \pm 0,02 \\
1,25 \pm 0,02 \\
1,26 \pm 0,02 \\
1,25 \pm 0,02 \\
1,25 \pm 0,02 \\
1,24 \pm 0,04 \\
1,26 \pm 0,02 \\
1,26 \pm 0,02 \\
1,26 \pm 0,03 \\
1,26 \pm 0,03 \\
1,24 \pm 0,03 \\
1,26 \pm 0,02 \\
1,26 \pm 0,03 \\
1,26 \pm 0,03 \\
1,26 \pm 0,03 \\
1,26 \pm 0,03\end{array}$ & $\begin{array}{c}0 \\
+0,01^{-} \\
-0,02^{-} \\
0 \\
+0,01^{-} \\
-0,01^{-} \\
+0,02^{-} \\
-0,01^{-} \\
+0,01^{-} \\
0 \\
-0,01^{-} \\
-0,02^{-} \\
-0,02^{-} \\
+0,01^{-} \\
-0,01^{-} \\
-0,01^{-} \\
-0,01^{-} \\
-0,01^{-} \\
+0,01^{-}\end{array}$ \\
\hline
\end{tabular}


TAB. 6. - Ausbildung adulter Kastenmerkmale nach Umbettu Völker im Vergleich mit reinen Arbeiterinnen gleicher

TABL. 6. - Formation des caractères adultes de caste apr d'ouvrières de colonies orphelines et comparais de portions de couvain

\begin{tabular}{|c|c|c|c|c|c|c|c|c|c|}
\hline 1 & 2 & 3 & 4 & \multicolumn{3}{|c|}{5} & \multicolumn{2}{|c|}{6} & \\
\hline \multirow{5}{*}{$\begin{array}{l}\text { Vers. } \\
\text { Nr. }\end{array}$} & \multirow{5}{*}{$\begin{array}{l}\text { Alter der } \\
\text { umgebette- } \\
\text { ten König. } \\
\text { Maden } \\
\text { Age des } \\
\text { larves de } \\
\text { greffees }\end{array}$} & \multirow{5}{*}{$\begin{array}{c}\text { Vers. } \\
\text { Tiere } \\
\mathrm{n}_{\mathrm{a}} \\
\\
\text { Insectes } \\
\text { testés } \\
\mathrm{n}_{a}\end{array}$} & \multirow{5}{*}{$\begin{array}{c}\text { Vergl. } \\
\text { Arb. } \\
\mathrm{n}_{\mathrm{b}} \\
\text { Ouvrières } \\
\begin{array}{c}\text { comparées } \\
\mathrm{n}_{h}\end{array}\end{array}$} & \multicolumn{3}{|c|}{ Mandibelindex } & \multirow{4}{*}{\multicolumn{2}{|c|}{$\begin{array}{c}\text { Kaukante } \\
\text { in \% 2n } \\
\text { (Vers. Tiere) } \\
A \quad \ddot{U} A \\
\text { Arêtes } \\
\text { mandibulaires } \\
\text { en \% } 2 \mathrm{n} \\
\text { (Ins. testés) }\end{array}$}} & \multirow{5}{*}{\begin{tabular}{|cr}
\multicolumn{2}{|c|}{ Vers. } \\
$\bar{x}$ \\
Insectes test \\
$\bar{x}$
\end{tabular}} \\
\hline & & & & \multirow{4}{*}{ 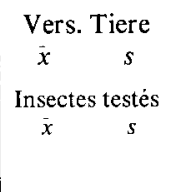 } & \multirow{4}{*}{$\begin{array}{c}\text { Vergl. Arb. } \\
\dot{x} \quad s \\
\oint_{\bar{x}} \text { compar. } \\
s\end{array}$} & \multirow{4}{*}{$\begin{array}{c}\text { Differenz } \\
a-b \\
\text { Diff. } \\
a-b\end{array}$} & & & \\
\hline & & & & & & & & & \\
\hline & & & & & & & & & \\
\hline & & & & & & & & $\ddot{U} A$ & \\
\hline 1 & $3 / 4-1$ & 20 & 24 & $4,95 \pm 0,18$ & $4,93 \pm 0,15$ & $+0,02^{-}$ & 100 & - & $1,79 \pm 0,0$ \\
\hline 2 & $1 \quad-11 / 2$ & 22 & 26 & $4,96 \pm 0,20$ & $4,97 \pm 0,18$ & $-0,01^{-}$ & 100 & - & $1,79 \pm 0$, \\
\hline 3 & $11 / 4-11 / 2$ & 10 & 24 & $4,88 \pm 0,19$ & $4,93 \pm 0,15$ & $-0,05^{-}$ & 100 & - & $1,79 \pm 0$, \\
\hline 4 & & 28 & 27 & $4,92 \pm 0,15$ & $4,90 \pm 0,16$ & $+0,02^{-}$ & 98 & 2 & $1,77 \pm 0$, \\
\hline 5 & $11 / 2-11 / 4$ & 19 & 27 & $4,99 \pm 0,19$ & $4,99 \pm 0,19$ & 0 & 100 & - & $1,79 \pm 0$, \\
\hline 6 & & 22 & 21 & $4,93 \pm 0,23$ & $4,92 \pm 0,18$ & $+0,01^{-}$ & 100 & - & $1,78 \pm 0$, \\
\hline 7 & $13 / 4-2$ & 21 & 27 & $5,02 \pm 0,16$ & $4,99 \pm 0,19$ & $+0,03^{-}$ & 100 & - & $1,79 \pm 0$ \\
\hline 8 & & 18 & 27 & $4,92 \pm 0,17$ & $4,90 \pm 0,16$ & $+0,02^{-}$ & 97 & 3 & $1,79 \pm$ \\
\hline 9 & $-21 / 4$ & 16 & 17 & $4,98 \pm 0,22$ & $4,94 \pm 0,15$ & $+0,04^{-}$ & 100 & - & $1,78 \pm$ \\
\hline 10 & & 16 & 21 & $4,91 \pm 0,25$ & $4,92 \pm 0,18$ & $-0,01^{-}$ & 100 & - & $1,78 \pm 0$ \\
\hline 11 & $21 / 4-21 / 2$ & 9 & 24 & $4,87 \pm 0,15$ & $4,93 \pm 0,15$ & $-0,06^{-}$ & 100 & - & $1,78 \pm$ \\
\hline 12 & $21 / 2-23 / 4$ & 17 & 26 & $4,93 \pm 0,17$ & $4,97+0,18$ & $-0,04^{-}$ & 100 & - & $1,78 \pm 0$ \\
\hline 13 & & 14 & 24 & $4,99 \pm 0,19$ & $4,93 \pm 0,15$ & $+0,06^{-}$ & 100 & - & $1,77 \pm 0$ \\
\hline 14 & $23 / 4-3$ & 14 & 22 & $4,93 \pm 0,20$ & $4,93 \pm 0,15$ & 0 & 82 & 18 & $1,78 \pm 0$ \\
\hline 15 & $\begin{array}{ll}3 & -31 / 4\end{array}$ & 13 & 21 & $5,00 \pm 0,25$ & $4,92 \pm 0,18$ & $+0,08^{-}$ & 100 & - & $1,79 \pm 0$ \\
\hline 16 & & 4 & 19 & $4,84 \pm 0,23$ & $4,91 \pm 0,16$ & $-0,07^{-}$ & 50 & 50 & $1,77 \pm 0$ \\
\hline 17 & & 11 & 19 & $4,81 \pm 0,19$ & $4,91 \pm 0,16$ & $+0,10^{-}$ & 82 & 18 & $1,75 \pm 0$ \\
\hline 18 & $31 / 4-31 / 2$ & 3 & 19 & $4,95 \pm 0,15$ & $4,91 \pm 0,16$ & $+0,04^{-}$ & 100 & - & $1,79 \pm 0$ \\
\hline 19 & $31 / 2-33 / 4$ & 3 & 22 & $4,98 \pm 0,20$ & $4,93 \pm 0,15$ & $+0,05^{-}$ & 67 & 33 & $1,72 \pm 0$ \\
\hline
\end{tabular}


schieden alter Königinnenlarven in Arbeiterzellen weiselrichtiger nft aus benachbarten Brutbezirken (Statistik siehe Tab. 5).

transfert de larves de reines d'âge variable dans des cellules c des ouvrières pures de même origine provenant

es (statistiques voir Tabl. 5).

\begin{tabular}{|c|c|c|c|c|c|c|c|c|c|}
\hline \multicolumn{2}{|l|}{7} & \multicolumn{2}{|c|}{8} & \multicolumn{2}{|c|}{9} & \multicolumn{2}{|c|}{10} & \multicolumn{2}{|c|}{11} \\
\hline \multicolumn{2}{|l|}{ Fersenindex } & \multirow{2}{*}{\multicolumn{2}{|c|}{$\begin{array}{c}\text { Bürste } \\
\text { in K1. \% } \\
\text { (V. Tiere) } \\
A \quad \ddot{U} A \\
\text { Brosse } \\
\text { en \% classe } \\
\text { (Insectes } \\
\text { testés) }\end{array}$}} & \multicolumn{2}{|c|}{$\begin{array}{c}\text { Kamm } \\
\text { in Kl. \% } \\
\text { (V. Tiere) }\end{array}$} & \multicolumn{2}{|c|}{$\begin{array}{l}\text { Körbchen } \\
\text { in Kl. \% } \\
\text { (V. Tiere) }\end{array}$} & \multicolumn{2}{|c|}{$\begin{array}{l}\text { Ungef. Entwicklungs- } \\
\text { dauer in Tagen } \\
\text { Durée de développement en jours }\end{array}$} \\
\hline $\begin{array}{l}\text { Vergl. Arb. } \\
\qquad \bar{x} \quad s \\
Q_{\bar{x}} \text { compar. }\end{array}$ & $\begin{array}{c}\text { Differenz } \\
a-b \\
\text { Diff. } \\
a-b\end{array}$ & & & \multicolumn{2}{|c|}{$\begin{array}{c}A \quad U A \\
\text { Peigne } \\
\text { en \% classe } \\
\text { (Insectes } \\
\text { testés) }\end{array}$} & \multicolumn{2}{|c|}{$\begin{array}{c}A \quad \ddot{U} A \\
\text { Corbeille } \\
\text { en \% classe } \\
\text { (Insectes } \\
\text { testés) } \\
A \quad \ddot{U} A\end{array}$} & $\begin{array}{c}\text { Vers. Tiere } \\
\text { Insectes testés }\end{array}$ & $\begin{array}{l}\text { Vergl. Arb. } \\
\text { Q compar. }\end{array}$ \\
\hline $\begin{array}{l}1,78 \pm 0,04 \\
1,79 \pm 0,05 \\
1,78 \pm 0,04 \\
1,78 \pm 0,03 \\
1,78 \pm 0,04 \\
1,78 \pm 0,03 \\
1,78 \pm 0,04 \\
1,78 \pm 0,01 \\
1,78 \pm 0,05 \\
1,78 \pm 0,03 \\
1,78 \pm 0,04 \\
1,79 \pm 0,05 \\
1,78 \pm 0,04 \\
1,78 \pm 0,05 \\
1,78 \pm 0,03 \\
1,79 \pm 0,02 \\
1,79 \pm 0,03 \\
1,79 \pm 0,02 \\
1,78 \pm 0,05\end{array}$ & $\begin{array}{l}+0,01^{-} \\
0 \\
+0,01^{-} \\
-0,01^{-} \\
+0,01^{-} \\
0 \\
+0,01^{-} \\
+0,01^{-} \\
0 \\
0 \\
0 \\
-0,01^{-} \\
-0,01^{-} \\
0 \\
+0,01^{-} \\
-0,02^{-} \\
-0,04^{-} \\
0 \\
-0,06^{-}\end{array}$ & $\begin{array}{r}100 \\
100 \\
100 \\
100 \\
100 \\
100 \\
100 \\
100 \\
100 \\
100 \\
100 \\
100 \\
100 \\
100 \\
100 \\
100 \\
82 \\
100 \\
67\end{array}$ & $\begin{array}{l}- \\
- \\
- \\
- \\
- \\
- \\
- \\
- \\
- \\
- \\
- \\
- \\
- \\
- \\
- \\
- \\
18 \\
- \\
33\end{array}$ & $\begin{array}{r}100 \\
100 \\
100 \\
100 \\
100 \\
100 \\
100 \\
100 \\
100 \\
100 \\
100 \\
100 \\
100 \\
100 \\
100 \\
100 \\
91 \\
100 \\
67\end{array}$ & $\begin{array}{l}- \\
- \\
- \\
- \\
- \\
- \\
- \\
- \\
- \\
- \\
- \\
- \\
- \\
- \\
- \\
- \\
9 \\
- \\
33\end{array}$ & $\begin{array}{r}100 \\
100 \\
100 \\
100 \\
100 \\
100 \\
100 \\
100 \\
100 \\
100 \\
100 \\
100 \\
100 \\
100 \\
100 \\
100 \\
100 \\
100 \\
67\end{array}$ & $\begin{array}{l}- \\
- \\
- \\
- \\
- \\
- \\
- \\
- \\
- \\
- \\
- \\
- \\
- \\
- \\
- \\
- \\
- \\
- \\
33\end{array}$ & $\begin{array}{l}\text { ca. } 20 \\
201 / 4 \\
20 \\
193 / 4 \\
201 / 4 \\
20 \\
20 \\
201 / 4 \\
20 \\
193 / 4 \\
193 / 4 \\
201 / 2 \\
201 / 4 \\
201 / 4 \\
191 / 2 \\
191 / 2 \\
20 \\
19 \\
20\end{array}$ & $\begin{array}{l}\text { ca. } 20 \\
20 \\
20 \\
20 \\
193 / 4 \\
20 \\
193 / 4 \\
20 \\
20 \\
20 \\
20 \\
20 \\
20 \\
193 / 4 \\
20 \\
20 \\
20 \\
20 \\
193 / 4\end{array}$ \\
\hline
\end{tabular}


1964) lassen sich sämtliche Versuche zum Gewichtsvergleich gemeinsam auswerten. Es errechnete sich für die Gewichtsüberlegenheit der Imagines aus Königinnenlarven eine Irrtumswahrscheinlichkeit von $\mathrm{p}=0,00096$.

3. Was die Ovarien anlangt, findet man in Spalte 6 unter den Versuchstieren schon bei einem Umlarvalter von 2 - 2 1/4 Tagen vereinzelt Individuen mit vermehrten Eischläuchen (Maximumwerte!). Nach der Umbettung von 2 1/2 - 2 3/4-tägigen Larven lässt sich der Unterschied in den Ovariolenzahlen zu den Vergleichstieren (in einem Fall) bereits statistisch absichern. Allerdings zeigen die in der Tabelle angegebenen Minimumwerte, dass auch nach der Umbettung relativ alter Maden immer wieder Imagines mit arbeitermässigen Ovariolen auftraten. Sie waren sogar meist in der Überzahl.

4. Überraschenderweise ist die Vesicula seminalis bei allen Versuchstieren - un geachtet des Umlarvalters der Königinnenmaden - arbeiterinnenmässig ausgebildet (Spalte 7). Auch Einzeltiere mit deutlich erhöhten Ovariolenzahlen hatten arbeiterhafte Samenblasen. Ob die mittlere Sicherung der Differenz zwischen den Samenblasendiametern der Versuchs- und Vergleichstiere in Versuch Nr. 17 eine erste Tendenz zur Samenblasenvergrösserung bei den Versuchstieren andeutet, ist bei Berücksichtigung des Gesamtbildes der Versuchsreihe fraglich. Der Durchmesser königlicher Samenblasen beträgt um 45 Teilstriche.

5. Die mittleren Indexwerte des Kopfes (Spalte 8). der Mandibeln und Metatar sen (Tab. 6, Spalte 5 und 6) sind bei Versuchs- und Vergleichstieren ohne kastenspezifischen Unterschied. Das gilt bezeichnender weise auch noch bei der Umbettung über 3 Tage alter Königinnenlarven. Die etwas spitzere Kaukante der Mandibel einzelner Individuen und geringfügig vom Arbeitertyp abweichende Hinterbeine ergeben äusserlich noch keine Zwischentiere.

6. Auch die Entwicklungszeiten der Versuchstiere unterschieden sich kaum von denen der zu vergleichenden Arbeitsbienen (Spalte 11). Die Tiere in den Versuchsund Kontrollwabenstücken schlüpften etwa zur gleichen Zeit zwischen 19 und $201 / 2$ Tagen nach der Eiablage. Es gab in beiden Fällen ausnahmsweise "zu früh " und "verzögert " schlüpfende Individuen. Insgesamt schlüpften die Tiere relativ früh. Man sollte aber bedenken, dass sie während ihrer ganzen Puppenzeit im Brutschrank lebten, wo eine gleichmässige Temperatur von $35 \pm 1{ }^{\circ} \mathrm{C}$ herrschte. Temperaturschwankun gen im Bienenvolk schaffen ungünstigere Verhältnisse.

3. - Hungerversuche mit decklungsreifen Königinnenmaden

\section{a) Überblick}

In Tabelle 7 sind die Überlebensverhältnisse von 50 gleichzeitig in 2 Pflegevölkern aufgezogenen Königinnenlarven wiedergegeben, nachdem sie im Streckmadenstadium aus ihren Zellen und dem Volk genommen wurden. Sie waren bei der Überfüh- 
ТАВ. 7. - Übersicht über die Hungerversuche mil Königinnen-Allmaden.

TABL. 7. - Tableau synoptique des expériences de faim avec de vieilles larves de reines.

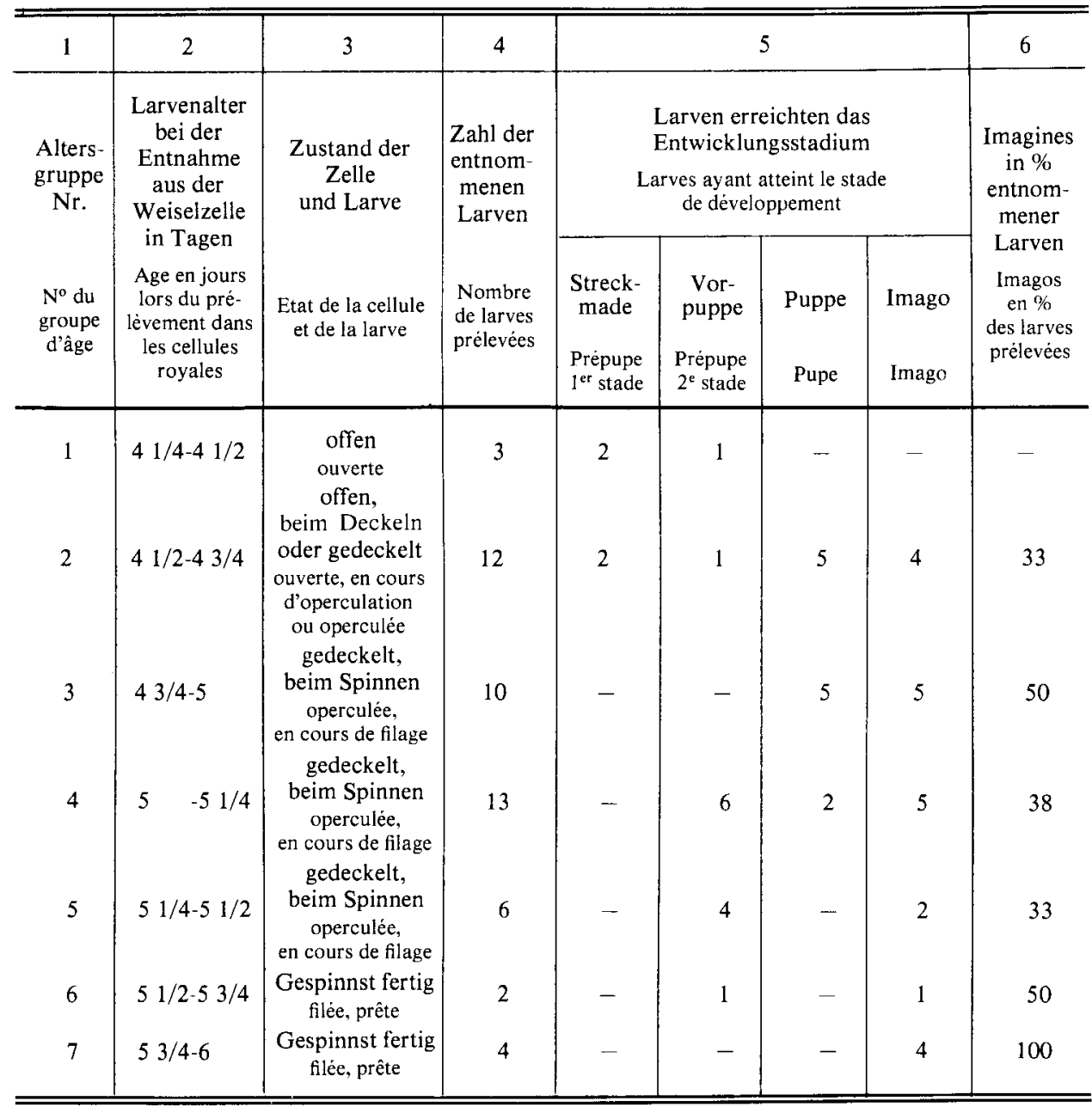

rung in den Brutschrank zwischen 4 und $61 / 2$ Tage alt. Die jüngsten Maden stammten aus noch offenen Zellen. Die Deckelung fand im Alter von $41 / 2$ bis $43 / 4$ Tagen statt. Alle später entnommenen Maden befanden sich bereits in verdeckelten Zellen. Insgesamt gingen 29 Tiere während der Weiterentwicklung im Brutschrank zugrunde. Die Verluste traten hauptsächlich auf dem Vorpuppen- und Puppenstadium auf. Wenn man die Maden nach Abschluss ihrer Spinntätigkeit aus den Zellen nahm, gab es keine Ausfälle mehr.

\section{b) Ergebnisse}

Ich habe alle überlebenden Tiere aus den Hungerversuchen einzeln in Tabelle 8 erfasst. Jede Zeile gehört einem Versuchstier. Die Imagines wurden nach dem ungefäh- 
TAB. 8. - Ausbildung adulter Kastenmerkm Königinnenmad

TABL. 8. - Formation des caractères adu aux larves de reine dont

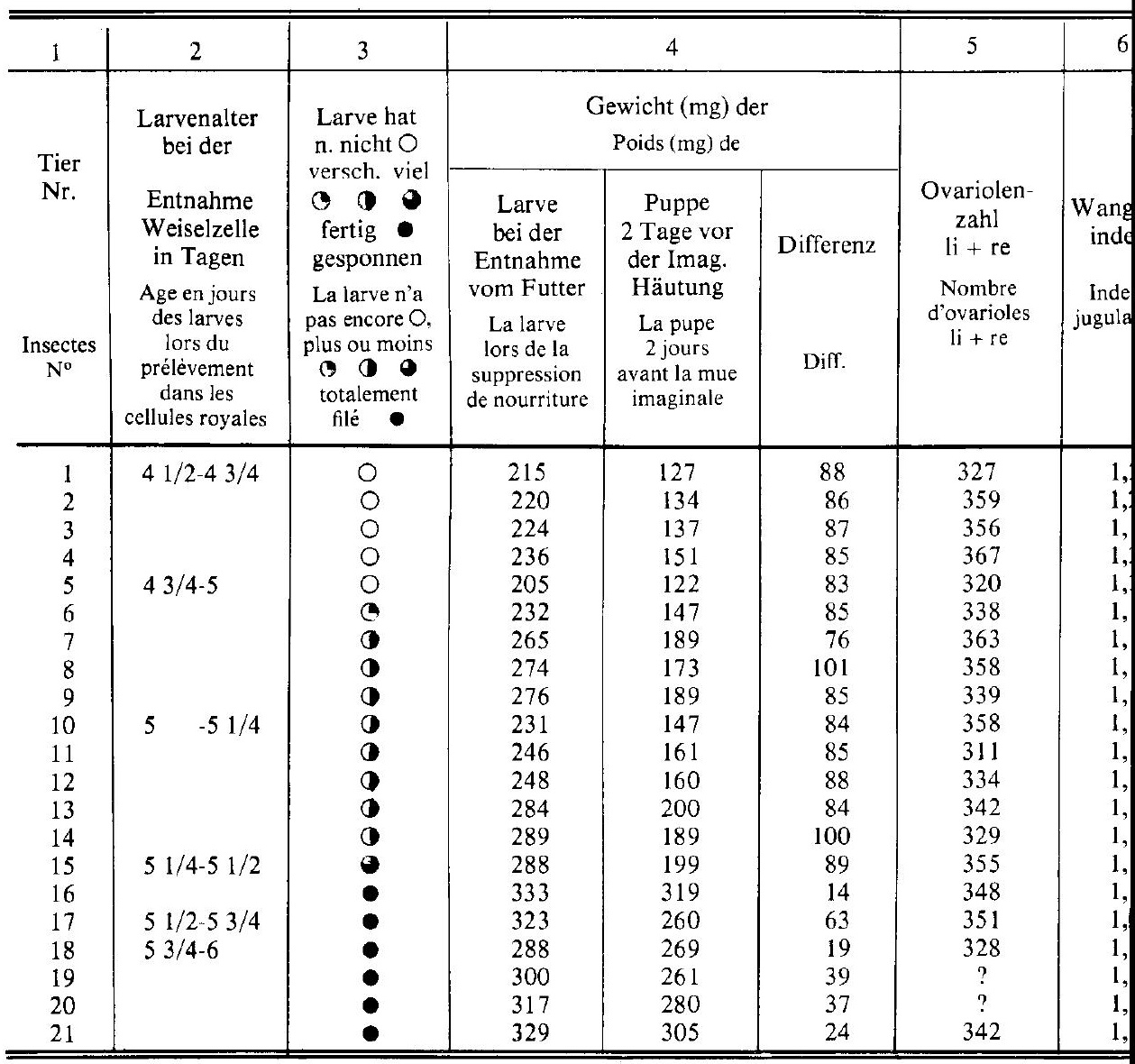


ren Alter der Streckmade bei der Entnahme aus der Weiselzelle und - innerhalb dieser Altersstufe - nach dem Larvengewicht geordnet. Dabei interessierte mich besonders, ob und wieviel die Larven beim Herausnehmen aus der Zelle bereits gesponnen hatten. Den Umfang der vorausgegangenen Spinntätigkeit konnte man daran abschätzen, wie weit das Gespinnst in der Zelle zum Futtersaft reichte, und wie dicht es unter der Zellkappe war. Die weitere Spinntätigkeit der Larve in ihren "Verpuppungsschiffchen" half den Befund abzusichern (Spalte 3).

Aus der Tabelle ist zu entnehmen :

1. Die Differenz zwischen Larven- und Puppengewicht (Spalte 4) ist bei den älteren Hungerlarven geringer als bei den jüngeren. Sie setzt sich aus der Aufzehrung von Körpermasse während des Einspinnens der hungernden Larven und dem Gewichts-

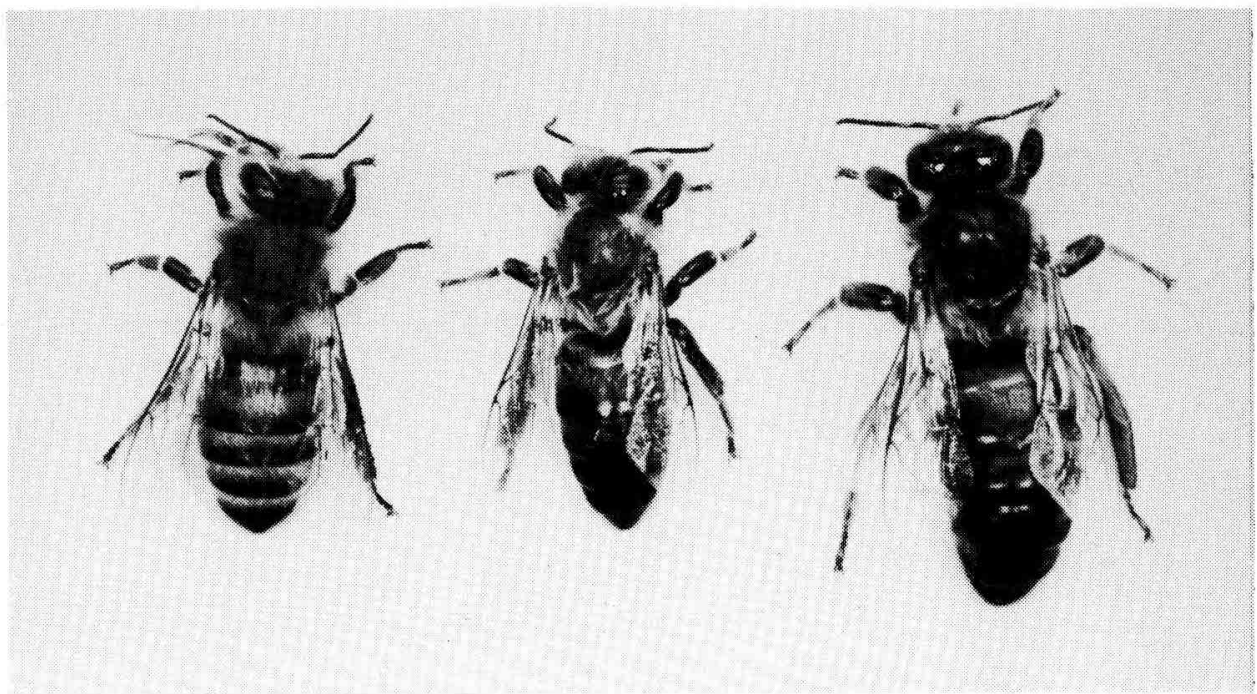

Aвв. 3. - Das Bild illustriert die Grössenverhältnisse einer normalen Arbeitswie e (links), einer normalen Königin (rechts) und einer a Hungerkönigin 》 (Mitte).

FIG. 3. - La figure illustre les rapports de taille d'une ouvrière normale (à gauche), d'une reine normale (à droite) et "d'une reine famélique " (au milieu).

schwund während der Puppenruhe zusammen. Die älteren Larven aus der Tabelle (Nr. 16-21) haben aber nicht gehungert. Erstaunlich ist ihr unterschiedlicher Gewichtsverlust. Schon in einer früheren Arbeit konnte ich nachweisen, dass in der Regel schwerere Puppen stärker an Gewichr abnahmen als leichte (WEIss 1967). Aber auch der Gewichtsschwund beim Einspinnen der Larven scheint grossen Schwankungen unterworfen zu sein. Aus dem Zahlenmaterial der Tabelle kann man ihn sehr überschlagsweise mit 50 bis $70 \mathrm{mg}$ annehmen.

2. Die jüngsten Königinnen-larven, welche sich ohne Futter weiter-entwickelten, waren $4 \frac{1 / 2}{-4} 3 / 4$ Tage alt. 
3. Je früher die Larven aus den Zellen entnommen wurden und je weniger sie bereits gesponnen hatten, desto mehr wichen sie - soweit sie sich zum fertigen Tier weiterentwickelten - in ihren äusseren Merkmalen vom Typ des Vollweibchens ab. Solange sie spannen und Nahrung aufnahmen, waren sie offenbar noch nicht vollkommen ausdeterminiert.

4. Übergangstiere waren immer besonders klein, was nicht ausschloss, dass auch relativ königinnenhafte Tiere mitunter sehr klein ausfielen, z.B. Nr. 6 und Abb. 3. Typische Königinnen präsentieren sich in Nr. 16 - 21. Ihre Larven hatten bei Versuchsbeginn die Spinnarbeit bereits beendet.

5. Die Ovariolenzahl wird durch die Hungerzeit während des Einspinnens nicht mehr beeinflusst (Spalte 5 ).

6. Trotz der nahezu reinen Übergangsformen, die aus sehr jungen Hungertieren entstanden, war die Imaginalhäutung gegenüber typischen Königinnen nur wenig, d.h. nur einen Tag verzögert.

\section{DISKUSSION}

Arbeiterlarven und Königinnenlarven sind während ihrer ersten Lebenstage aufgrund von Fütterungsfaktoren voll in die jeweils andere weibliche Bienenkaste umstimmbar, Während dieser Zeit sind die Larven entwicklungsneutral. Man spricht von der bipotenten oder sensiblen Larvenphase.

Von allen untersuchten kastentypischen Kriterien scheint nur das erreichbare Imaginalgewicht eine Ausnahme zu machen. Wie besonders aus den Umbettungsversuchen von Arbeitermaden in Weiselwiegen hervorgeht, nimmt das Gewicht der fertigen Tiere schon $a b$ frühem Entwicklungsstadium mit steigendem Umbettungsalter laufend ab, was bereits Weaver (1959), JoRDAN (1960), WoyKe (1971) und WeIss (1974) wussten. Dass sich bei der Umbettung von Königinnenmaden verschiedenen Alters in Arbeiterinnenzellen der Sachverhalt mit den Adultgewichten nicht einfach umkehrt, dürfte am begrenzten Rauminhalt der Arbeiterzellen liegen. Unter diesen Umständen ist es schon erstaunlich, dass in der Mehrzahl der Versuche aus den in Arbeiterzellen aufgezogenen Königinnenmaden Tiere hervorgingen, die deutlich schwerer waren als die vergleichsweise in der Nachbarschaft geschlüpften Arbeiterinnen. Auch hier reagierten schon relativ junge Königinnenmaden in diesem Sinn.

Damit zeigen die Umbettungsversuche von weiblichen Bienenlarven verschiedener Kastenzugehörigkeit, dass für das kastenspezifische Wachstum des weiblichen Bienenwesens die Larvennahrung bereits auf jüngstem Entwicklungsstadium bedeutsam ist. Die besonders frühe und empfindliche Reaktion des Adultgewichts bei der ernährungsbedingten Kastenumstellung der weiblichen Bienenlarven weist auf eine Ausnahmestellung des Gewichts unter den Kastenmerkmalen hin. Während alle morphologischen Vorgänge der Kastenentwicklung über hormonale Zwischenschaltungen ablaufen, ist es nicht ausgeschlossen, dass das Adultgewicht zusätzlich oder aus- 
schliesslich unmittelbar über die Beschaffenheit des Futters (z.B. den Nährstoffgehalt) beeinflusst wird. Erstaunlich ist nur, dass diese frühen Entwicklungsunterschiede gewichtsmässig bis in das imaginale Alter nachwirken. Ich habe schon früher, in Anbetracht der experimentell erstellbaren Riesenarbeiterinnen und Zwergköniginnen darauf hingewiesen, dass man das Adultgewicht vielleicht gar nicht als echtes Kastenkriterium ansehen sollte (WEISS 1974).

Die vorliegenden Versuche bestätigen die Feststellung BecKers (1925), nämlich den raschen Verlust der bipotenten Entwicklungsfähigkeit der Arbeiterlarven nach dem 3. Larventag. Auf die sensible Entwicklungsphase folgt eine kritische Phase, in der die Plastizität der Kastenbildung abnimmt. Bei den Königinnenlarven finden sich erste Anzeichen hierfür schon etwas früher als bei der Arbeiterlarve. Allerdings beschränken sie sich vollkommen auf die Ausbildung des Ovars. Königlich angepflegte Larven ergeben bereits bei Umbettung im Alter von 2 1/2 Tagen Imagines, die gegenüber typischen Arbeiterinnen vermehrte Ovariolen besitzen. Trotzdem gibt es auch unter Tieren aus älteren Königinnenmaden, deren Ovariolenzahlen immer näher an die Verhältnisse bei der Königin herankommen, immer wieder Einzelexemplare. die reine Arbeiterovarien aufweisen.

Die frühe Beeinflussbarkeit der Ovariolenzahl scheint eine Besonderheit der Kastenentwicklung der Königinnenlarve gegenüber der der Arbeitermade zu sein. Nach Löschel (1916) nimmt die Ovariolenzahl der Königinnenlarve bis zum 2. Larventag rasch und danach " nicht mehr nennenswert " zu. Der Autor meint, dass die endgültige Zahl der Ovariolen am 5. Larventag erreicht sei. Zu dieser Zeit zählte er in einer Ovarhälfte 155 Eischläuche. MEIER (1916) glaubte, bei der Arbeiterinnenlarve bis zum 2. Lebenstag in der Ovarentwicklung keinen Unterschied zur Königinnenmade festzustellen. In den nächsten Tagen würde die Zahl der Eischläuche jedoch etwas langsamer als bei der Königinnenmade ansteigen. Er zählte am 5. Larventag einseitig 130 Ovariolen. Während der vorpupalen Ruhezeit nach dem Einspinnen der Made vollzieht sich dann bei der Arbeiterin die Rückbildung der Ovariolenzahl auf die bleibende Restmenge. Die Samenblase wächst dagegen bis zum Ende der Vorpuppenzeit weiter und wird erst in den ersten Puppentagen zurückgebildet. Nach diesen Feststellungen darf man bei Umbettungsversuchen mit Königinnen- und Arbeiterinnenmaden nach dem 2. Larventag mit einer Beeinflussung der Ovarentwicklung durch die vorausgegangene kastentypische Pflege rechnen. In unseren Versuchen war sie jedoch nur bei Königinnenmaden nachweisbar, bei Arbeitermaden trat sie erst bei einem Umlarvalter von 3 Tagen in Erscheinung. Interessanterweise haben jüngste histologische Untersuchungen von Dogra, UlRICH und REMBold (1977) entgegen früheren Feststellungen von CANetTi u. M. (1964) und RitCey u. Dixon (1969) bei Königinnenlarven bereits während des 2. Larventages (Ende der 2., Anfang der 3. Zwischenhäutungsphase) ein stärkeres Wachstum der Nervenfortsätze der Neurosekretorischen Zellen mit Andeutung des Chiasmas erkennen lassen, während die Chiasmabildung bei Arbeiterlarven erst im Laufe des 3. Larventages (Anfang der 4. Zwischenhäutungsphase) sichtbar wurde. Ein kastenspezifischer Unterschied im histologischen Bild der Corpora allata trat erst während des 3. Larventages (Ende der 3. Zwi- 
schenhäutungsphase) auf, was im grossen und ganzen durch Befunde von LuKoschus (1956), Canetti u. M. (1964), Wang u. Shuel (1965), Ritcey u. Dixon (1969) und WIRTs (1973) bestätigt wird. Das Drüsenvolumen der Corpora allata nimmt bei der Königinnenlarve stärker zu als bei der Arbeiterlarve und es zeigen sich Strukturänderungen, die bei der Arbeiterlarve verzögert ablaufen. Insgesamt ist nach Dogra u. M. (1977) die Ausbildung der neurosekretorischen Zellen und der Wachstumsbeginn der Corpora allata bei den Arbeiterinnenlarven gegenüber den Verhältnissen bei der Königinnenmade um etwa einen Tag verzögert.

Es ist erstaunlich, dass in meinen Versuchen die Samenblase bei den Imagines aus königlich vor- und arbeitermässig weitergepflegten Tieren keine messbare Vergrösse rung gegenüber reinen Arbeiterinnen aufwies. Das gilt auch noch für die Umbettung relativ alter Königinnenmaden und vor allem für Tiere mit hohen Ovariolenzahlen. Eierstock und Samenblase reagierten also bei diesen Umbettungsversuchen nicht gleichsinnig. Umgekehert entstanden jedoch bei der Umbettung von Arbeiterlarven während ihrer kritischen Phase in Königinnenzellen Imagines, die sowohl hinsichtlich der Ovariolenzahl als auch des Samenblasenvolumens kastentypische Abweichungen aufwiesen.

Wie beim Gewicht erscheint es auch bei der Entwicklung des reproduktiven Systems möglich, dass sie nicht nur hormonell gesteuert, sondern ausserdem noch direkt über Nahrungsfaktoren beeinflusst werden kann. Man erinnere sich nur an die Riesen arbeiterinnen VON RHEINS (1933) mit den vergrösserten Eierstöcken und Samenblasen. Er erzielte diese Thiere ausschliesslich durch Mästung mit besonders reichlichem Arbeitermischfutter. Diese selektive Empfindlichkeit der Ovarentwicklung bei der Aufzucht von Königinnenmaden in Arbeiterzellen deutet im übrigen auf eine besonders leichte Beeinflussbarkeit dieses Kastenmerkmals durch Aussenfaktoren hin.

Überraschenderweise haben sich die sekundären Kastenmerkmale der Kopfform, der Mandibelausbildung und der Beschaffenheit des Pollensammelbeins bei der Umbettung von Königinnenlarven bis zu 3 3/4 Tagen in Arbeiterzellen durch die königliche Vorversorgung fast überhaupt nicht beeinflussen lassen. Gelegentliche kleine Abweichungen an der Mandibel und, noch seltener, am Sammelbein lagen doch stets sehr nahe an der Arbeiterinnengrenze. Auch die Entwicklungsdauer der Versuchstiere war gegenüber den Vergleichsbienen nicht erkennbar verkürzt.

Der Zeitfaktor ist ein Kastenkriterium besonderer Art. Zwar besteht zwischen der Entwicklungszeit der Königin mit 16 Tagen und der Arbeiterin mit 21 Tagen (von der Eibablage bis zum Schlupf gerechnet) ein deutlicher Unterschied, doch können beträchtliche Schwankungen nach oben und unten vorkommen. Von grossem Einfluss ist dabei die Entwicklungstemperatur (Himmer 1932; Buchner 1953, Soose 1954). Echte Zwischentiere zeigen in der Regel gegenüber Königinnen eine verlängerte Entwicklungszeit, aber es ist bekannt, dass auch typische Königinnen anormal lange Entwicklungszeiten aufweisen können. Das beweist sehr eindrucksvoll eine Versuchsreihe mit fortgesetzter Königinnenaufzucht im unverjüngten Volk (WEISS 1972). Die aus den späteren Serien gewonnenen typischen Königinnen brauchten zu 
ihrer Entwicklung fast ebensolange wie Arbeitsbienen. Nicht selten findet man auch in normalen Zuchtserien gut gepflegte Zellen, die verzögert gedeckelt werden und deren Königinnen bis zu 2 Tage später schlüpfen. Hierbei massgebliche Fütterungsanomalien müssen nicht undedingt etwas mit der Kastenspezifität der Ernährung zu tun haben.

Wenn bei der Umbettung von Königinnenmaden in Arbeiterzellen trotz der besonders frühen Beeinflussung der Ovariolenausbildung alle anderen Kastenkriterien sogar besonders lange umstimmbar zu bleiben scheinen, darf man folgendes nicht übersehen :

1. Dem Wachstum der königlichen Larve ist durch die Enge der Arbeiterzelle eine empfindliche Grenze gesetzt. Es ist nicht ausgeschlossen, dass hieraus ein hemmender Einfluss auf die Durchschlagsfähigkeit von königlichen Merkmalen erwächst. Trotz häufiger Versuche habe ich leider nicht vermocht, Königinnenmaden in Drohnenzellen weiselrichtiger Völker zur Entwicklung zu bringen. Selbst wenn ich die Maden mitten zwischen gleichaltrige Drohnenbrut plazierte, sorgten die Bienen für ihr rasches Verschwinden.

2. Verdächtig sind die grossen Ausfälle bei der Umbettung von über 3 Tage alten Königinnenmaden in Arbeiterzellen. Obwohl es nicht ganz einfach ist, grössere Maden in Arbeiterzellen umzubetten, kann man die geringe Annahme der Larven doch nicht einfach auf die Möglichkeit einer mechanischen Schädigung zurückführen. Die Maden wurden sogar sehr häufig noch gedeckelt und verschwanden trotzdem bald danach. Es schien auch, als ob sie vorzeitig gedeckelt worden wären. Vielleicht sind sie besonders schnell gewachsen oder sie verhielten sich anders als Arbeitermaden. Die Deckelung erfolgte stets in der für Arbeiterbrut typischen Weise. Wenn die Zellen danach ausgeräumt wurden, kann das damit zusammenhängen, dass die Insassen verhungert sind. Aber auch bei der Umstimmung von Arbeitermaden in die königliche Entwicklungsrichtung gab es in der kritischen Phase besonders grosse Ausfälle. Es ist nicht ausgeschlossen, dass sich gerade unter den verschwundenen Maden Übergangsformen befunden haben, die uns auf diese Weise nicht zu Gesicht kamen. In beiden Fällen fragt es sich auch, ob die Bienen, welche die vordeterminierten Larven nicht in Pflege nahmen, die Diskrepanz zwischen Larvenwiege und Kastenzugehörigkeit ihres Inhalts erkannten und die Larven deshalb entfernten, oder ob nur das abweichende Verhalten der Maden in der ihnen fremden Umgebung an ihrem Verschwinden schuld war.

Während wir bei der Umbettung von Arbeitermaden in Weiselbehälter in der kritischen Phase eine Reihe - auch in den Aussenmerkmalen - typischer Zwischenformen erzielt haben, fehlen uns solche bei den Umstimmungsversuchen mit Königinnenlarven. Wir können hier also nicht genau sagen, wann die bipotente Phase in die kritische Phase mit Einfluss der kastenspezifischen Futterversorgung auch auf die äusseren königlichen Merkmale und die Entwicklungszeit übergeht. Wir dürfen nach unseren Versuchsergebnissen, insbesondere unter Berücksichtigung der Hungerversuche mit spinnreifen Königinnenlarven, aber annehmen, dass dieser Zeitpunkt um die Mitte des 5. Larventages eintritt. Ich habe in Abb. 4 versucht, die voneinander abweichenden Verhältnısse der Kastenumstimmung bei Arbeiterinnen- und Königinnenlarven schematisch darzustellen. 
MECHANIK DER KASTENENTSTEHUNG

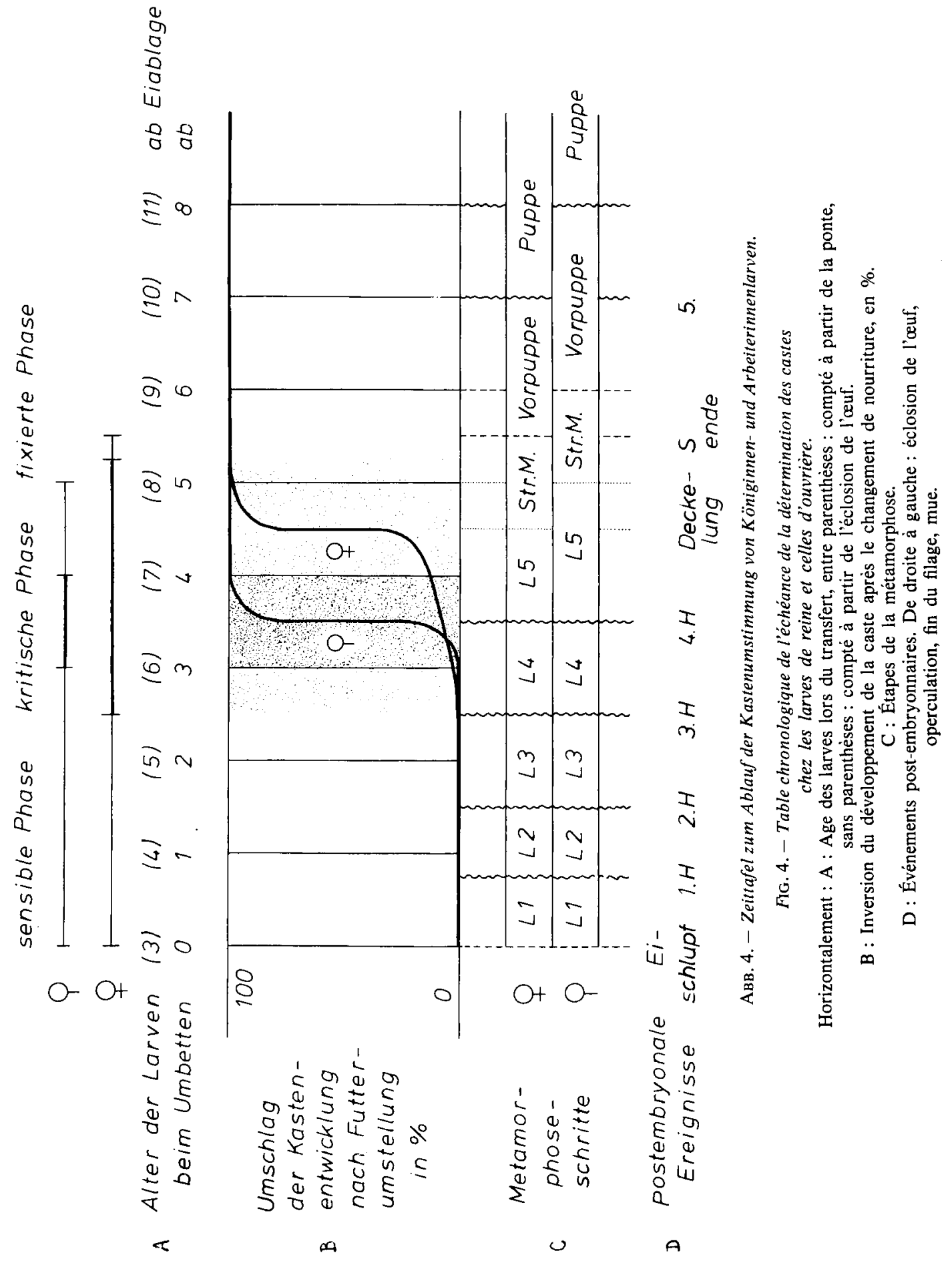


Unsere Umbettungsversuche mit Arbeiterlarven verschiedenen Alters in Königin nenzellen weisen deutlich aus, dass bei der Arbeiterinnenentwicklung auf die kritische Phase eine fixierte Phase folgt, in der keine Beeinflussung der Kastenbestimmung durch weitere Nahrungsaufnahme mehr möglich ist. Diese Phase währt noch mindestens 1 Tag.

Völlig anders liegen die Verhältnisse bei der Königinnenmade. Unsere Hungerversuche mit älteren Königinnenlarven zeigen, dass hier die determinierende Wirkung des Futters bis kurz vor Ende der Nahrungsaufnahme durch die Larve anhält. Die Königinnenmade, welche erst mit Abschluss der Spinnarbeit das Fressen einstellt, war 6 Stunden vor diesem Zeitpunkt noch nicht völlig ausdeterminiert. Die äusseren Kastenmerkmale, insbesondere die Ausbildung der Mandibeln und der Hinterbeine, waren noch phänotypisch im Sinne der Kastenumwandlung beeinflussbar. Dazu bedurfte es nicht einmal der Endversorgung mit Futter der anderen Kaste, d.h. Mischfutter älterer Arbeiterinnenlarven - es genügte schon der Mangel jedweden Futters. Das bedeutet, dass die determinierende Wirkung des Königinnenfutters nahezu solange andauert, als die Made Nahrung aufnimmt. Wenn die Larve nicht bis zum Ende ihrer Spinnzeit fressen kann, ist mit der Entstehung nicht nur kleinerer sondern auch in den Aussenmerkmalen unvollkommener Geschlechtstiere zu rechnen.

Das Einzige, was sich bei totalem Futtermangel am Ende der Fressperiode nicht mehr ändert, ist die Ovariolenzahl. Wir wissen aus den Untersuchungen MEIER's (1916), dass sich die auch in der Arbeiterkaste zunächst zahlreich angelegten Ovariolen erst nach der Fressperiode während der Vorpuppenzeit zurückbilden. Die Königinnenmade, welche erst gegen Ende ihrer Fresszeit durch eine Hungerperiode in arbeiterähnliche Verhältnisse gerät, ist dazu nicht mehr in der Lage.

Einer besonderen Bemerkung bedarf das unerwartete Auftreten einiger Versuchstiere aus der Gruppe 1-1 1/4-tägig umgelarvter Arbeitermaden, welche im fertig entwickelten Zustand deutliche Abweichungen vom reinen Königinnentypus zeigten. Ich habe schon darauf hingewiesen, dass das Pflegevolk der Cordovan-Mutante angehörte. Es nahm trotz ansehnlicher Stärke nur sehr wenige Zellen an. Nach diesem Misserfolg wurde das Volk aus dem Versuch herausgenommen, bekam aber interesseshalber noch einmal eine Zuchtserie mit etwa 1 Tag alten Larven. Unter dem äusserst spärlichen Aufzuchtergebnis befanden sich wieder ein paar Zwischenformen.

Diese Beobachtung ist nicht einmalig. Ich bin im Laufe jahrelanger Zuchtversuche schon öfter mit einem gestörten Aufzuchtgeschehen dieser Form konfrontiert worden. Der Cordovan-Typ des Pflegevolkes ist dabei nicht das Wesentliche. In meinen Versuchen über die Fähigkeit alternder Bienen zur Weiselaufzucht (WEISS 1972) arbeitete ich mit 2 Carnica-Völkern. Es gelang mir einmal 17 und einmal 20 Zuchtserien unmittelbar hintereinander von den gleichen, zuletzt wenigstens 97- bzw. 112-tägigen Bienen aufziehen zu lassen. Gegen das Ende der Versuchsreihe traten immer mehr Tiere mit deutlichen Abweichungen vom reinen Königinnentyp auf - wenn auch einzelne Individuen bis zuletzt mit Ausnahme des Gewichtes als eindeutige Königinnen anzusprechen waren. Je mehr die Pflegekapazität der alternden Ammenbienen ab- 
nahm, desto grösser wurde der Prozentsatz an Abweichungen. Hier war zweifellos das Alter der Pflegebienen entscheidend. Im Falle des Cordovan-Volkes war es wahrscheinlich die Pflegeuntüchtigkeit dieser Mutante. In anderen Fällen kann so etwas wie eine volkseigentümliche, rassenunabhängige Pflegeunlust vorliegen. Festzuhalten ist, dass die Entstehung nicht nur kleinerer, sondern auch in ihren Kastenkriterien unvollkommen ausgebildeter Königinnen nicht ausschliesslich durch die Verwendung überalteter Aufzuchtmaden, sondern auch durch mangelhafte Pflege seitens der sie versorgenden Ammenbienen verursacht sein kann.

Wir können zusammenfassend feststellen : Die Determination zur einen oder anderen Kaste des weiblichen Bienenwesens findet nicht zu einem bestimmten Zeitpunkt der Larvenentwicklung statt. Sie vollzieht sich kontinuierlich über einen langen Entwicklungsabschnitt. Die Arbeiterlarve ist bis zum Ende ihres 4. Lebenstages, die Königinnenlarve fast bis ans Ende ihrer Fressperiode kastenspezifisch beeinfluss- und wandelbar. Allerdings sind die Larven zu verschiedenen Zeiten ihrer Entwicklung gegen determinierende Einflüsse verschieden empfindlich. Während sie anfänglich zu einer völligen Umkehr ihrer kastenspezifischen Entwicklung fähig sind (sensible Phase), nimmt diese Plastizität mit Eintreten der kritischen Phase rasch ab. Die kritische Phase liegt bei der Arbeiterlarve zwischen dem 3. und 4. Larventag, bei der Königinnenmade beginnt sie hinsichtlich der Ovariolenentwicklung schon etwas früher, während die übrigen Kastenmerkmale erst später betroffen werden. Bei der Arbeiterinnenlarve folgt nach dem 4 . Larventag eine wenigstens $1 \mathrm{Tag}$ währende, gegen determinierende Einflüsse völlig unempfindliche Endphase in der durch Nahrungsaufnahme gekennzeichneten Larvenentwicklung. Diese " fixierte Phase " schrumpft bei der Königinnenmade, welche bis fast ans Ende der Spinnperiode um die Mitte des 6. Larventages determinierbar bleibt, auf wenige Stunden zusammen.

Es bedarf kaum der Anmerkung, dass mit der morphologischen Determination eine physiologische verbunden ist - und ihr vorausgeht. So hat man bei Königinnenund Arbeiterinnenlarven schon vor der kritischen Phase stoffwechselphysiologische und innersekretorische Unterschiede verschiedener Art nachgewiesen. Aber bezeichnenderweise vermögen diese Verschiedenheiten keinen Einfluss auf die Ausprägbarkeit der adulten Merkmale während der sensiblen Phase auszuüben (s. Lit. bei WEISS 1971, 1975). Die Natur scheint das Bienenvolk möglichst lange in die Lage versetzen zu wollen, aus Arbeiterbrut funktionsfähige Ersatzköniginnen nachzuziehen. 


\section{RÉSUME \\ MÉCANISME DE LA FORMATION DES CASTES CHEZ L'ABEILLE}

(Apis mellifica L.)

1) La larve femelle d'Abeille traverse dans les premiers jours de sa vie une phase de développement bipotentielle ou sensible, durant laquelle elle a la possibilité de se développer soit en reine typique, soit en ouvrière, selon les soins prodigués par les nourrices. Seul le poids des adultes est influencé par une alimentation basée depuis le début de la vie sur la nourriture de l'autre caste.

2) La phase sensible n'a pas la même durée chez la larve de reine et celle d'ouvrière. La larve d'ouvrière peut encore s'inverser totalement en reine jusqu'à la fin du 3ème jour. La phase critique, durant laquelle le développement antérieur laisse des traces irréversibles lors de l'inversion, commence le 4ème jour. Il se développe pendant cette période des formes de passage vers l'ouvrière. Tous les critères spécifiques des castes s'insèrent dans ce schéma, excepté le poids de l'adulte qui peut être influencé vers une caste déterminée dès le début du développement larvaire. Il n'est pas certain qu'il faille reconnaitre le poids de l'adulte comme un véritable caractère de caste.

Jusqu'à l'âge d'environ 2 jours et demi les larves de reine peuvent se transformer à $100 \%$ en larves d'ouvrières par modification de la nourriture. En conséquence, le développement antérieur est rendu perceptible chez les individus par la formation de nombreux ovarioles chez l'adulte. Au fur et à mesure qu'augmente l'âge du transfert, l'influence sur les ovaires se fait plus marquante. Curieusement les répercussions sur les autres critères de caste sont faibles - mème après un transfert de larves royales âgées de plus de 3 jours. Des individus avec des arêtes mandibulaires un peu plus pointues et une patte fortement modifiée en vue de la récolte ont peu d'importance. Il est particulièrement frappant que, malgré un nombre fortement élevé d'ovarioles, les spermathèques conservent un caractère d'ouvrière. De même la durée de développement des larves n'est pas réduite. Par conséquent il n'y a pas formation, comme c'est le cas lorsqu'on transfère des larves d'ouvrières entre le 3ème et le 4ème jour de vie, d'individus intercastes extérieurement typiques. La phase critique pour les caractères externes commence environ 1 jour plus tard chez les larves de reine que chez celles des ouvrières.

3) La phase critique, au cours de laquelle se perd la bipotentialité de développement des larves femelles, est caractérisée par une forte mortalité de ces insectes. Dans la mesure où les larves d'ouvrières âgées disparaissent après le transfert hors des cellules royales, cette mortalité peut ètre due à leur comportement anormal dans un environnement qui ne leur est pas adapté. Mais une partie de ces insectes restent fixés au stade de prépupe ou de pupe.

Les larves de reines plus âgées greffées dans les cellules d'ouvrières disparaissent aussi en grand nombre juste après le transfert. Lorsqu'elles sont operculées, le moment de l'operculation se situe souvent trop tôt par rapport à l'âge des larves. Les abeilles nettoient ces cellules très vite après que les larves operculées sont probablement mortes de faim. Si nous ne trouvons aucun intercaste, cela peut être dû au fait que ces individus ont été victimes d'un développement perturbé en raison d'un taux de mortalité élevé.

4) Le transfert dans les cellules royales de larves d'ouvrières âgées de 4 jours et plus donne naissance - dans la mesure où celles-ci se développent - à des ouvrières typiques. Le fait pour les ouvrières d'ingérer de la nourriture royale ne peut plus exercer d'influence déterminante sur les imagos qui vont en résulter. Au moins un jour avant la cessation de prise de nourriture, qui se termine avec l'operculation de la cellule, la larve d'ouvrière est déjà si fortement fixée à sa caste que le développement en cours ne peut plus subir aucune déviation (phase fixée).

Les conditions sont tout autres chez la larve de reine. Celle-ci est encore influençable jusqu'à la fin de la période d'alimentation (= période de filage), environ un jour après l'operculation de la cellule royale. Cela signifie que durant toute cette période il est possible de modifier les critères de caste en direction de la caste ouvrière. Pour cela il n'est pas nécessaire de changer le type de nourriture, mais simplement de la retirer. La phase fixée se réduit par conséquent à quelques heures pour la larve de reine (voir fig. 4).

5) Des imagos possédant des caractères royaux défectueux se sont exceptionnellement développés dans l'une de nos expériences lors du transfert de larves d'ouvrières âgées de un jour à un jour un quart. Il 
ne s'agit pas dans ce cas de l'irruption d'une prédétermination larvaire et ouvrière, mais il convient plutôt d'attribuer le développement anormal à une insuffisance de soins de la part d'une colonie éleveuse de mauvaise qualité. Des soins d'élevage médiocres peuvent donc induire des reines imparfaites.

\section{LITERATUR VERZEICHNIS}

BUCHNER R., 1953. - Beeinflussung der Grösse der Arbeitsbiene durch Raum - und Nahrungsmangel während der Larvenzeit. Roux' Arch. Entwickl.mech. Org. 146, 444-579.

CANETti S. J., Shuel R. W. u. Dixon S. E. - Studies in the mode of action of royal jelly in honey bee development. IV. Development within the brain and retrocerebral complex of female honeybee larvae. Can. J. Zool. 42, 229-233.

Dogra G. S., Ulrich G. M. u. Rembold H., 1977. - A comparative study of the endocrine system of the honeybee larvae under normal and experimental conditions. Z. Naturforsch. 32 c, 637-642.

DöNHOFF E., 1859. - Über die künstliche Entstehung von Zwergköniginnen. Eichst. Bz. 15 (1), 8-9.

ECKERT J. E., 1934. - Studies in the number of ovarioles in queen honeybees in relation to body size. $J$. econ. Ent. 27, 629-635.

GontarsKı H., 1941. - Über Zwischenformen zwischen Königin und Arbeiterin im Staat der Honigbiene (Apis mellifica). Z. wiss. Zool. 154 (3) 345-356.

HAYDAK M. H., 1943. - Larval food and development of castes in the Honeybee. J. econ. Ent. 36 (5), 778 792.

HAYDAK M. H., 1968. - Nutrition des larves d'abeilles. In Chauvin : Traité de biologie de l'abeille I. 202333, Masson et Cie, Paris.

Himmer A., 1932. - Die Temperaturverhältnisse bei sozialen Hymenopteren. Biol. Rev. 7, 224-253.

JAY S. C., 1964. - Starvation studies of larval honey bees. Can. J. Zool. 42, 455-462.

Johansson R. S. K. u. Johansson M. P., 1958. - Royal jelly II. Bee World 39, 254-264, 277-286.

JoRDAN R., 1960. - Die Zucht der Königin ausgehend vom Ei. Bienenvater 81 (1) 3-7.

KLEIN J., 1925. - Futterbrei und weibliche Bienenlarve. Die Bienenpfl. 26, H. 5 (1904) ref. bei Zander u. Becker Erl. Jb. Bienenkde. 3, 161-246.

Löschel F., 1916. - III. Die postembryonale Entwicklung des Geschlechtsapparates der Bienenkönigin (Apis mellifica L.) Z. angew. Ent. 3 (1) 21-44.

Lukoschus F., 1956. - Untersuchungen zur Entwicklung der Kastenmerkmale bei der Honigbiene (Apis mellifica L.). Z. Morph. Ökol. Tiere 45, 157-197.

MeIER K., 1916. - IV. Die postembryonale Entwicklung des Geschlechts-apparates der Arbeitsbiene (Apis mellifica L.) Z. angew. Ent. 3 (1) 45-65.

Nelson J. A. u. Sturtevant A. P., 1924. - Growth and feeding of honeybee larvae. Bull. U. S. Dept. Agr. Nr. 1222, 1-24.

Rembold H. 1964. - Die Kastenentstehung bei der Honigbiene (Apis mellifica L.) Naturwiss. 51 (3) 4954.

Rembold H., 1974. - Die Kastenbildung bei der Honigbiene, Apis mellifica L., aus biochemischer Sicht. In Sozialpolymorphismus bei Insekten. Wiss. Verlagsges. Stuttgart S. 350-403.

RheIN W. von, 1933. - Über die Entstehung des weiblichen Dimorphismus im Bienenstaate. Wilh. Roux'Arch. Entwickl.mech. Org. 129, 601-665.

RitCEy G. M. u. Dixon S. E., 1969. - Postembryonic development of the endocrine system in the female honeybee castes, Apis mellifera L. Proc. ent. Soc. Ont. 100, 124-138.

Shuel R. W. u. Dixon S. E., 1960. - The early establishment of dimorphism in the female honeybee, Apis mellifera L. Inc. soc. 7 (3) 265-282.

Shuel (R. W. u. Dixon S. E., 1973. - Regulatory mechanisms in caste development in the honeybee, Apis mellifera L. Proc. VII. intern. Congr. IUSSI London. S. 349-356. 
SMITH M. V., 1959. - Queen differenciation and the biological testing of royal jelly. Mem. Cornell Univ. Agr. Exp. Sta. Nr. 356.

Soose E., 1954. - Einfluss der Temperatur auf die Ausgestaltung von Flügelgeäder und Panzerfarbe der Honigbiene. Arch. Bienenkde, 31, 49-66.

Townsend A. F. u. Shuel R. W. 1962. - Some recent advances in apicultural research. Ann. Rev. Ent. 7, 481-500.

VAGT W. 1955. - Morphologische Untersuchungen an Nachschaffungsköniginnen von Apis mellifica, die aus verschieden alten Larven gezüchtet wurden. Z. Bienenf. 3, 73-80.

VeHLich A. V., 1930. - Entwicklungsmechanische Studien an Bienenlarven. Z. wiss. Zool. 136 (2) 210 222.

WANG D. J. u. SHUEL R. W. 1965. - Studies in the mode of action of royal jelly in honeybee development. V. The influence of diet on ovary development. J. apic. Res. 4, 149-160.

WeAver N., 1957. - Effect of larval age on dimorphic differentiation of the female honeybee. Ann. ent. Soc. Amer. 50 (3) 283-294.

Weber Erna. Grundriss der biologischen Statistik. 5. Aufl. Gustav Fischer, Jena, 1964.

WeIss K., 1962. - Versuche zur Methodik der Königinnenzucht aus dem Ei. Z. Bienenf. 6 (2) 37-47.

WeIss K., 1967 a. - Zur vergleichenden Gewichtsbestimmung von Bienenköniginnen. Z. Bienenf. 9 (1) 1 21.

WEISs K., 1967 b. - Über den Einfluss verschiedenartiger Weiselwiegen auf die Annahme und das Königinnengewicht in der künstlichen Nachschaffungszucht. Z. Bienenf. 9 (3) 121-134.

WEIss K., 1971. - Über die Ausbildung und Leistung von Königinnen aus Eiern und jungen Arbeitermaden. Apidologie 2 (1) 3-47.

WEISS K., 1972. - Verlauf und Beschaffenheit aufeinanderfolgender Zuchtserien im unverjüngten Pflegevolk. Apiacta 7 (3) 110-114.

WEISs K., 1974. - Zur Frage des Königinnengewichtes in Abhängigkeit von Umlarvalter und Larvenversorgung. Apidologie 5 (2) 127-147.

WeIsS K., 1975 a. - Zur kastenspezifischen Ernährung der weiblichen Bienenlarve (Apis mellifica L.) Apidologie 6 (2) 95-120.

WeISs K., 1975 b. - Die Zucht aus dem Ei - ein Ausweg. Imkerfr. 30 (6) 170-173.

WIRTz P., 1973. - Differentiation in the honeybee larva. Meded. Landbouwhogeschool Wageningen (735), $1-55$.

WOYKE J., 1971. - Correlations between the age at which honeybee brood was grafted, characteristics of the resultant queens, and results of insemination. J. apic. Res. 10, 45-55.

ZANDER E. u. Becker, F. 1925. - Die Ausbildung des Geschlechtes bei der Honigbiene II. Erl. Jb. Bienenkde, 3, 161-246. 